\title{
A multi-omics investigation of the composition and function of extracellular vesicles along the temporal trajectory of COVID-19
}

\author{
Sin Man Lam ${ }^{1,2,10}$, Chao Zhang ${ }^{3,10}$, Zehua Wang ${ }^{1,4,10}$, Zhen Ni1,4,10, Shaohua Zhang ${ }^{1,10}$, \\ Siyuan Yang ${ }^{5,10}$, Xiahe Huang1, Lesong Mo ${ }^{1,4}$, Jie Li ${ }^{1,4}$, Bernett Lee ${ }^{6}$, Mei Mei', Lei Huang ${ }^{3}$, Ming Shi \\ Zhe $\mathrm{Xu}^{3}$, Fan-Ping Meng ${ }^{3}$, Wen-Jing $\mathrm{Cao}^{3,7}$, Ming-Ju Zhou ${ }^{3,7}$, Lei Shi ${ }^{3}$, Gek Huey Chua ${ }^{2}$, Bowen $\mathrm{Li}^{2}$, \\ Jiabao $\mathrm{Cao}^{4,8}$, Jun Wang ${ }^{8}$, Shilai Bao ${ }^{1,4}$, Yingchun Wang ${ }^{1,4}$, Jin-Wen Song ${ }^{3 凶}$, Fujie Zhang ${ }^{9 凶}$, \\ Fu-Sheng Wang $\mathbb{D}^{3 凶}$ and Guanghou Shui ${ }^{1,4}{ }^{\square}$
}

\begin{abstract}
Exosomes represent a subtype of extracellular vesicle that is released through retrograde transport and fusion of multivesicular bodies with the plasma membrane'. Although no perfect methodologies currently exist for the high-throughput, unbiased isolation of pure plasma exosomes ${ }^{2,3}$, investigation of exosome-enriched plasma fractions of extracellular vesicles can confer a glimpse into the endocytic pathway on a systems level. Here we conduct high-coverage lipidomics with an emphasis on sterols and oxysterols, and proteomic analyses of exosome-enriched extracellular vesicles (EVs hereafter) from patients at different temporal stages of COVID-19, including the presymptomatic, hyperinflammatory, resolution and convalescent phases. Our study highlights dysregulated raft lipid metabolism that underlies changes in EV lipid membrane anisotropy that alter the exosomal localization of presenilin-1 (PS-1) in the hyperinflammatory phase. We also show in vitro that EVs from different temporal phases trigger distinct metabolic and transcriptional responses in recipient cells, including in alveolar epithelial cells, which denote the primary site of infection, and liver hepatocytes, which represent a distal secondary site. In comparison to the hyperinflammatory phase, EVs from the resolution phase induce opposing effects on eukaryotic translation and Notch signalling. Our results provide insights into cellular lipid metabolism and inter-tissue crosstalk at different stages of COVID-19 and are a resource to increase our understanding of metabolic dysregulation in COVID-19.
\end{abstract}

As of early June 2021, COVID-19 has infected more than 1.7 billion people worldwide, with global deaths attributed to COVID-19 exceeding 3.7 million according to the World Health Organization (WHO) COVID-19 Dashboard (https://covid19.who.int/). Although COVID-19 is primarily an infectious disease caused by the SARS-CoV-2, the infection can trigger a myriad of metabolic perturbations that may alter the trajectory of the course of the disease and the final outcome. The temporal course of COVID-19 pathogenesis is marked by direct viral-mediated effects on host metabolism, as well as collateral damage, which results from the response of the host to the pathogen ${ }^{4}$. Originally perceived as a means for cells to excrete unwanted materials ${ }^{5}$, later studies have implicated exosomes in intercellular communication, particularly with respect to viral transmission ${ }^{6,7}$ and immune responses ${ }^{8}$. As lipid-bilayer-enclosed structures, EVs exhibit varying lipid membrane compositions depending on the nature and physiological status of their parent cells ${ }^{9}$. Severity-associated alterations in membrane lipid composition were reported for exosome-enriched EVs in COVID-19 (ref. ${ }^{10}$ ). As exosomes originate primarily from within the MVBs that concentrate more than half of the membrane cholesterol along the endocytic pathway ${ }^{11}$, cholesterol homeostasis elicits important effects on exosome biology and functions ${ }^{12}$. Cholesterol homeostasis is identified as a key host pathway that mediates SARS-CoV-2 infection of human cells ${ }^{13,14}$. Cholesterol with sphingomyelin (SM) also forms ordered raft membrane microdomains that fine-tune membrane fluidity and alter protein localization $^{15}$. For example, $\gamma$-secretase (GS), which cleaves NOTCH-1 to form Notch intracellular domains ${ }^{16}$ and $\beta$-amyloid precursor protein (APP) to form $\beta$-amyloid peptides $(\mathrm{A} \beta)^{17}$, displays preferential localization and enhanced activity in lipid rafts ${ }^{18}$. The lipid composition of EV membranes therefore can potentially alter the localization and activity of GS, modulating downstream pathways pathologically relevant to COVID-19.

We allocated plasma samples to four temporal stages on the basis of the sample collection date relative to the dates of hospital admission and discharge, SARS-CoV-2 RNA test results and associated laboratory findings (Table 1 and Supplementary Table 1; see Methods section 'Study design and participants' for details). No significant differences in baseline characteristics (age, sex and

'State Key Laboratory of Molecular Developmental Biology, Institute of Genetics and Developmental Biology, Chinese Academy of Sciences, Beijing, China. ${ }^{2}$ LipidALL Technologies Company Limited, Changzhou, China. ${ }^{3}$ Department of Infectious Diseases, Fifth Medical Center of Chinese PLA General Hospital, National Clinical Research Center for Infectious Diseases, Beijing, China. ${ }^{4}$ University of the Chinese Academy of Sciences, Beijing, China. ${ }^{5}$ Laboratory of Infectious Diseases Center, Beijing Ditan Hospital Capital Medical University, Beijing, China. ${ }^{6}$ Singapore Immunology Network, Agency for Science, Technology and Research (A`STAR), Singapore, Singapore. ${ }^{7}$ Department of Clinical Medicine, Bengbu Medical College, Anhui, China. ${ }^{8} \mathrm{CAS}$ Key Laboratory of Pathogenic Microbiology and Immunology, Institute of Microbiology, Chinese Academy of Sciences, Beijing, China. ${ }^{9}$ The Clinical and Research Center for Infectious Diseases, Beijing Ditan Hospital Capital Medical University, Beijing, China. ${ }^{10}$ These authors contributed equally: Sin Man Lam, Chao Zhang,

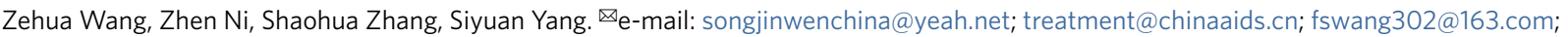

ghshui@genetics.ac.cn 
Table 1 | Demographics and baseline characteristics of patients with COVID-19

\begin{tabular}{|c|c|c|c|c|c|}
\hline & S1 & S2 & S3 & S4 & $P$ value \\
\hline$n$ & 16 & 25 & 19 & 18 & \\
\hline Age (years) (median (IQR)) & $41.00(36.75-52.25)$ & $39.00(29.00-46.00)$ & $35.00(30.50-50.50)$ & $45.00(39.50-49.50)$ & 0.351 \\
\hline Body mass index (median (IQR)) & $24.90(23.28-28.13)$ & $26.03(23.23-28.40)$ & $23.53(21.59-28.21)$ & $25.97(24.53-26.88)$ & 0.666 \\
\hline Clinical grade $(n(\%))$ & & & & & $0.427^{\mathrm{a}}$ \\
\hline Mild & $0(0.0 \%)$ & $10(40.0 \%)$ & $7(36.8 \%)$ & $3(16.7 \%)$ & \\
\hline Severe & $0(0.0 \%)$ & $5(20.0 \%)$ & $6(31.6 \%)$ & $5(27.8 \%)$ & \\
\hline SARS-CoV-2 positive $(n(\%))$ & $16(100.0 \%)$ & $25(100.0 \%)$ & $0(0.0 \%)$ & $0(0.0 \%)$ & $<0.001$ \\
\hline WBCs $\left(\times\left. 10^{9}\right|^{-1}\right)$ (median (IQR)) & $5.54(5.03-6.01)$ & $5.17(3.39-6.57)$ & $6.19(5.40-7.34)$ & $5.78(3.97-6.66)$ & 0.294 \\
\hline Haemoglobin $\left(\mathrm{gl}^{-1}\right)(\operatorname{median}(\mathrm{IQR}))$ & $150.00(129.25-155.75)$ & $147.00(134.00-154.00)$ & $138.00(125.50-152.50)$ & $121.00(109.25-141.75)$ & 0.021 \\
\hline $\begin{array}{l}\text { Platelets }\left(\times 10^{9} \mathrm{I}^{-1}\right) \text { (median (IQR)), } \\
\text { X10^9/L }\end{array}$ & $196.50(161.00-230.75)$ & $172.00(148.00-246.00)$ & $245.00(219.00-284.00)$ & $201.00(170.75-242.50)$ & 0.006 \\
\hline D-dimer (median (IQR)) & $0.24(0.13-0.36)$ & $0.24(0.15-0.57)$ & $0.27(0.16-1.02)$ & $0.64(0.19-0.71)$ & 0.411 \\
\hline Urea $($ mmoll-1) (median (IQR)) & $4.97(3.83-6.21)$ & $4.98(3.85-5.97)$ & $4.84(4.33-6.02)$ & $4.70(4.29-4.93)$ & 0.694 \\
\hline Creatinine $\left(\mu \mathrm{moll} \mathrm{I}^{-1}\right)(\operatorname{median}(\mathrm{IQR}))$ & $70.20(59.20-78.95)$ & $71.70(64.00-82.40)$ & $70.60(65.80-80.20)$ & $70.50(64.25-76.80)$ & 0.751 \\
\hline Uric acid $\left(\mu\right.$ mol I $\left.^{-1}\right)$ (median (IQR)) & $352.50(278.25-391.50)$ & $290.00(223.00-366.00)$ & $363.00(304.50-456.50)$ & $283.00(237.00-356.00)$ & 0.131 \\
\hline Glucose (mmoll-1) (median [IQR]) & $5.75(4.85-6.76)$ & $4.90(4.50-5.80)$ & $4.41(4.34-5.42)$ & $4.79(4.50-5.02)$ & 0.071 \\
\hline Bilirubin $\left(\mu \mathrm{mol} \mathrm{I}^{-1}\right)(\operatorname{median}(\mathrm{IQR}))$ & $10.10(7.83-11.98)$ & $10.50(8.50-13.50)$ & $11.80(9.65-13.40)$ & $9.40(8.30-10.57)$ & 0.143 \\
\hline Albumin $\left(\left.g\right|^{I^{-1}}\right)$ (median $\left.(I Q R)\right)$ & $42.35(40.77-45.33)$ & $43.00(40.00-45.20)$ & $42.70(40.25-44.30)$ & $38.50(36.25-41.75)$ & 0.13 \\
\hline $\begin{array}{l}\text { Alanine aminotransferase }\left(\mathrm{UI}^{-1}\right) \text { (median } \\
(\mathrm{IQR}))\end{array}$ & $21.90(16.75-25.57)$ & $33.00(16.30-65.00)$ & $32.70(19.10-59.05)$ & $34.50(26.05-42.62)$ & 0.193 \\
\hline Myoglobin (median (IQR)) & $17.00(11.00-24.22)$ & $21.00(13.00-22.94)$ & $12.00(10.00-22.00)$ & $21.00(11.00-21.00)$ & 0.581 \\
\hline $\begin{array}{l}\text { Erythrocyte sedimentation rate (median } \\
(\text { IQR)) }\end{array}$ & $12.50(6.75-17.25)$ & $17.00(8.00-33.00)$ & $20.00(10.00-43.00)$ & $34.00(14.00-53.00)$ & 0.135 \\
\hline Lymphocyte count (median (IQR)) & $\begin{array}{l}1,730.00 \\
(1,472.28-2,245.25)\end{array}$ & $\begin{array}{l}1,630.65 \\
(1,005.00-1,863.00)\end{array}$ & $\begin{array}{l}2,141.00 \\
(1,778.50-2,453.00)\end{array}$ & $\begin{array}{l}1,628.31 \\
(1,425.08-2,353.41)\end{array}$ & 0.01 \\
\hline T-cell frequency (median (IQR)) & $71.03(66.94-73.84)$ & $63.02(56.68-75.00)$ & $76.07(73.41-81.03)$ & $73.45(66.50-81.50)$ & 0.003 \\
\hline T-cell count (median (IQR)) & $1,233.50(874.75-1,550.75)$ & $\begin{array}{l}1,039.00 \\
(665.00-1,326.00)\end{array}$ & $\begin{array}{l}1,625.00 \\
(1,324.00-1,948.50)\end{array}$ & $\begin{array}{l}1,347.00 \\
(1,099.25-1,601.25)\end{array}$ & 0.001 \\
\hline CD8 frequency (median (IQR)) & $24.90(21.29-27.79)$ & $25.63(21.00-34.00)$ & $27.99(24.57-31.27)$ & $27.00(23.92-32.86)$ & 0.29 \\
\hline CD8 count (median (IQR)) & $406.50(298.00-581.75)$ & $438.00(266.00-532.00)$ & $607.00(488.50-738.50)$ & $464.00(389.75-650.75)$ & 0.016 \\
\hline CD4 frequency (median (IQR)) & $42.82(40.15-47.51)$ & $33.39(30.41-41.00)$ & $46.19(38.02-50.26)$ & $41.84(33.25-47.50)$ & 0.01 \\
\hline CD4 count (median (IQR)) & $699.50(576.00-1,057.75)$ & $503.00(330.00-747.00)$ & $872.00(712.50-1,072.50)$ & $670.50(558.25-949.50)$ & 0.004 \\
\hline CD4/CD8 ratio (median (IQR)) & $1.78(1.58-2.28)$ & $1.28(1.02-1.70)$ & $1.67(1.09-2.17)$ & $1.46(1.17-2.18)$ & 0.155 \\
\hline NK cell frequency (median (IQR)) & $11.50(7.50-23.75)$ & $18.00(12.00-24.00)$ & $10.00(6.00-12.00)$ & $12.50(9.00-20.25)$ & 0.027 \\
\hline NK cell count (median (IQR)) & $187.50(138.25-333.50)$ & $215.00(175.84-433.00)$ & $216.00(136.50-332.00)$ & $181.12(142.77-350.33)$ & 0.779 \\
\hline B-cell frequency (median (IQR)) & $13.00(8.94-17.50)$ & $12.00(8.00-18.00)$ & $11.00(7.88-13.00)$ & $10.50(7.25-13.75)$ & 0.247 \\
\hline B-cell count (median (IQR)) & $236.50(135.00-304.50)$ & $173.71(116.23-251.29)$ & $220.00(146.50-279.50)$ & $155.34(112.26-279.70)$ & 0.472 \\
\hline
\end{tabular}

Data are shown as medians and interquartile range (IQR) or $n$ and the percentage. $P$ values were computed using $\chi^{2}$ tests for categorical variables or two-sided Kruskal-Wallis tests for continuous variables. CD4, CD4 T cells; CD8, CD8 T cells; WBCs, white blood cells. ${ }^{2} P$ value on clinical grade was based on comparison among symptomatic S2, S3 and convalescent S4 patients excluding S1. Clinical grade classification was infeasible for presymtomatic $\$ 1$ patients who did not exhibit any clinical symptoms at the time of blood collection. 
body-massindex)wereobservedacrossthefourdiseasestages(Table 1). Slight coagulopathy was observed in the symptomatic, hyperinflammatory (S2) stage, indicated by a diminished platelet count and elevated levels of plasma lactate dehydrogenase. These observations corroborate previous clinical findings of appreciable coagulopathy in patients with COVID-19 early in the course of disease ${ }^{19-21}$. Consistent with findings from previous studies ${ }^{22}$, immune-cell profiles along the disease trajectory indicated a delayed and suppressed innate immune response to infection with SARS-CoV-2. Lymphocyte and T-cell counts were not reduced until the S2 stage, with accompanying hyperinflammation as evident from the sharp increase in C-reactive protein (Table 1). Clinical findings associated with patient blood samples supported our classification based on the disease trajectory of survivors of COVID-19 (Fig. 1a) into an immunosuppressed, presymptomatic (S1) stage followed by a symptomatic, hyperinflammatory (S2) stage to fight endogenous viral loads. The next parts of the disease trajectory comprising the resolution (S3) and convalescent (S4) stages become largely host driven (that is, SARS-CoV-2 RNA negative) and are characterized by a gradual resolution of inflammation (including the restoration of lymphocyte counts and inflammatory markers) (Table 1).

We used differential centrifugation followed by polymer-based precipitation to isolate exosome-enriched EVs from 78 temporal plasma samples of patients with COVID-19. The size distribution of the isolated EVs was verified by nanoscale flow cytometry analysis and was in agreement with the theoretical size of EVs $(<200 \mathrm{~nm})$, in which $>85 \%$ of EVs were particles $<180 \mathrm{~nm}$ and $>98 \%$ of EVs were particles $<300 \mathrm{~nm}$ (Fig. 1b,c). Isolated EVs were enriched in exosome-specific markers ${ }^{23}$, including TSG101, CD9 and CD63, relative to the supernatant and plasma (Extended Data Fig. 1a). Marker proteins for possible plasma contaminants (annexin A1 as a microvesicle marker; $\mathrm{ApoB}$ and $\mathrm{ApoE}$ as lipoprotein markers) were not appreciably detected compared with whole plasma samples with equal amounts of total protein loaded (Extended Data Fig. 1b,c). Transmission electron microscopy images of isolated EVs showed that the vesicles exhibited a cup-shaped vesicle morphology that is characteristic of exosomes with sizes around $100 \mathrm{~nm}$ (Extended Data Fig. 1d,e). These observations support that the isolated EVs were exosome enriched.

Using high-performance liquid chromatography-tandem mass spectrometry (HPLC-MS/MS), we quantitatively profiled the lipidome of exosome-enriched EVs, with emphasis on various oxidized derivatives of cholesterols and phytosterols known to elicit immuno-modulatory ${ }^{24}$ and antiviral ${ }^{25}$ effects. Quantified lipid levels were normalized to the sum of total membrane lipids (that is, molar fractions to total membrane lipids, MFM). A global clustering heat map was constructed using $\log _{2}$-transformed fold-changes and line plots were drawn on the basis of $z$ scores that illustrate the temporal patterns of changes in the EV lipidome during the course of COVID-19 (Fig. 2). Glycosylated sphingolipids such as globotriaosylceramides with very long-chain fatty acyls (that is, C22-C24), which have been reported to increase the susceptibility of $\mathrm{CD}^{+}{ }^{+} \mathrm{T}$ cells to HIV infection ${ }^{26}$, were specifically reduced in the convalescent S4 stage compared with the presymptomatic S1 stage (Fig. 2, box (1)). Numerous plasmalogen phosphatidylethanolamines (PEps) comprising polyunsaturated fatty acyls were specifically elevated in the presymptomatic S1 stage (Fig. 2, box (2)).
PEps were found to enrich in HIV particles ${ }^{27}$ and attenuate cholesterol biosynthesis by facilitating squalene monooxygenase degradation $^{28}$. Impeded cholesterol biosynthesis compromises the defence of the host against SARS-CoV-2 infection ${ }^{13}$. Indeed, the Reactome pathway 'cholesterol biosynthesis' was significantly downregulated in HepG2 cells treated with S1 EVs relative to EVs from healthy control individuals (Supplementary Table 2; described further below). Sulfatides, which are major constituents of myelin sheath and pancreas $\beta$-cells ${ }^{29}$, were reduced in EVs from the symptomatic S2 and S3 stages, but (partially) restored in the convalescent S4 stage (Fig. 2, box (3) and Extended Data Fig. 2a). Sulfatides regulate autoimmune processes ${ }^{29}$ and modulate insulin release from pancreatic islets to prevent $\beta$-cell exhaustion ${ }^{30}$. A previous case report and in vitro studies have suggested that SARS-CoV-2 could trigger insulin-dependent diabetes and has the potential to infect pancreatic $\beta$-cells ${ }^{31,32}$. Distinct increases in EV phosphatidylcholines (PCs) (Figs. 2, box (4), 3a-c) and phosphatidylserines (Figs. 2, box (5), $3 a-c)$ were observed in the resolution S3 and hyperinflammatory S2 stages, respectively. In a proinflammatory milieu, the phosphatidylserine content of EVs from vascular cells increases to promote calcification and coagulation $^{33}$. This agrees with the reduction in platelet count (that is, coagulopathy) that was found in S2 (Table 1).

Several oxysterols and sterols were elevated in both S2 and S4 EVs (Fig. 2, box (6)), indicating dysregulated cholesterol homeostasis on a systems level. Free cholesterol, several sterols as well as oxidized cholesterol derivatives were markedly reduced in S1 compared with S2 (Fig. 3a,b and Extended Data Fig. 3a). Broadly perceived as antiviral compounds that impede viral replication ${ }^{34}$, S1-specific reductions in oxysterols corroborate a clinical status of ongoing viral replication and a blunted immune response. For example, 27-hydroxycholesterol, which was recently shown to inhibit the replication of two human coronaviruses (SARS-CoV-2 and $\mathrm{HCoV-OC43)} \mathrm{in} \mathrm{vitro}{ }^{25}$, was particularly reduced in S1 EVs. Notably, 6-keto- $5 \alpha$-hydroxycholesterol and $3 \beta$-hydoxy-5-cholestenoic acid represent the only cholesterol derivatives analysed in this study that were significantly increased in S1 compared with S2 (Fig. 3b). 6-keto$5 \alpha$-hydroxycholesterol, which is also known as 6-oxo-cholestan$3 \beta, 5 \alpha$-diol, is involved in the regulation of inflammation and the immune response ${ }^{24,35}$. 6-keto- $5 \alpha$-hydroxycholesterol inhibits natural killer (NK) cell-mediated cytotoxicity ${ }^{36}$ and impedes cytolytic activity of $\mathrm{CD}^{+} \mathrm{T}$ lymphocytes ${ }^{37}$. As important components of innate immunity ${ }^{22}$, disrupting the cytolytic activities of these cells is expected to contribute to the delayed immune response and impeded virus clearance in S1. Furthermore, $3 \beta$-hydroxy-5-cholestenoic acid is a GS modulator ${ }^{38}$. Relative to non-steroidal GS modulators, GS modulators with steroid backbones possess appreciable inhibitory activity to Notch substrates in addition to inhibiting the cleavage of $\mathrm{APP}^{39}$. As 3 $\beta$-hydroxy-5-cholestnoic acid was specifically reduced in the S2 phase (Fig. 3b), we postulate that GS-mediated activity may vary during the course of COVID-19. Marked increases in various oxysterols were evident in S2 EVs compared with S1, including $5 \alpha, 6 \alpha$-epoxy-cholesterol, $6 \alpha$-hydroxy- $5 \alpha$-cholestanol, $7 \alpha$-hydroxycholestenone, 7-keto-cholesterol, cholesta-4,6-dien3-one, 24S,25-epoxy-cholesterol and dihydro-testis-meiosis activating sterol (dihydro-T-MAS) (Fig. 3b). General increases in oxysterols could have resulted from the overall state of oxidative stress and hyperinflammation that characterized S2. Lathosterol,

Fig. 1 | Investigating the EV composition along the temporal course of COVID-19. a, The temporal trajectory of COVID-19 is generally divided into four stages in survivors, which includes the presymptomatic (S1), hyperinflammatory (S2), resolution (S3) and convalescent (S4) phases. The first half of the trajectory (that is, SARS-CoV-2 RNA positive) is largely virus driven, characterized by immunosuppression and the response of the host to the invading pathogen, whereas the second half comprises mainly host-driven metabolism to resolve the hyperinflammation and restore physiological homeostasis. Exosome-enriched EVs were isolated from fasting plasma, and high-coverage analyses of exosomal lipidome with a focus on sterols and oxysterols were conducted. $\mathbf{b}$, The particle size distribution of isolated EVs. $n=4$ independent experiments. c, Particle sizes of isolated EVs analysed by nanoscale flow cytometry. Representative plot from $n=4$ independent experiments. 


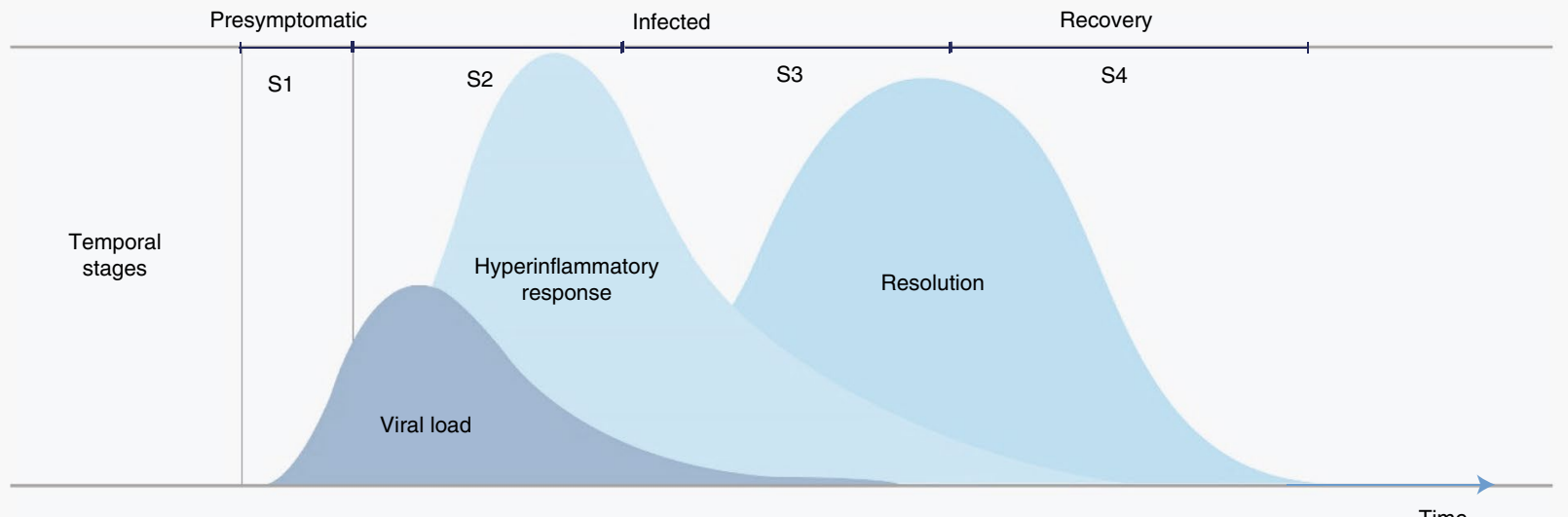

Time
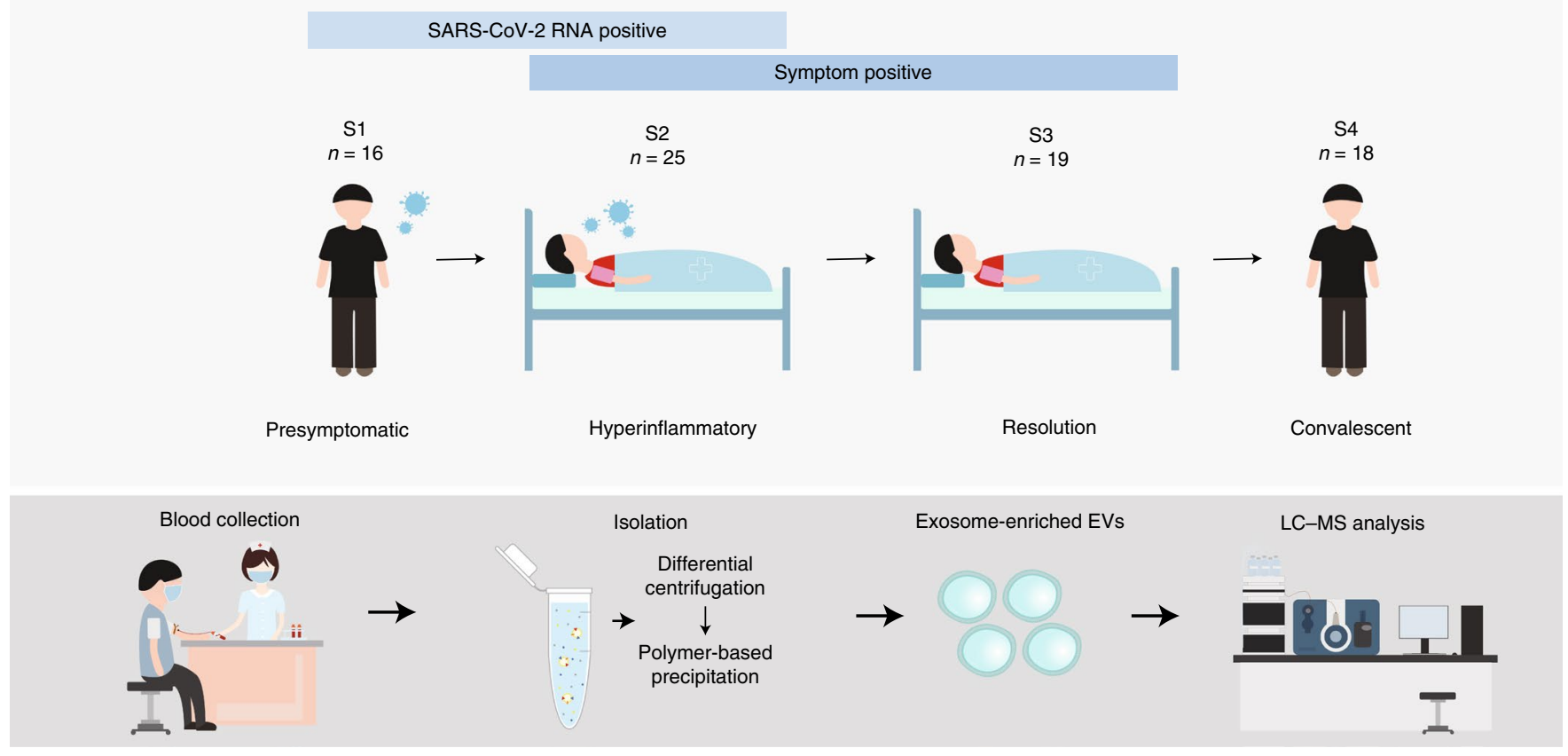

b

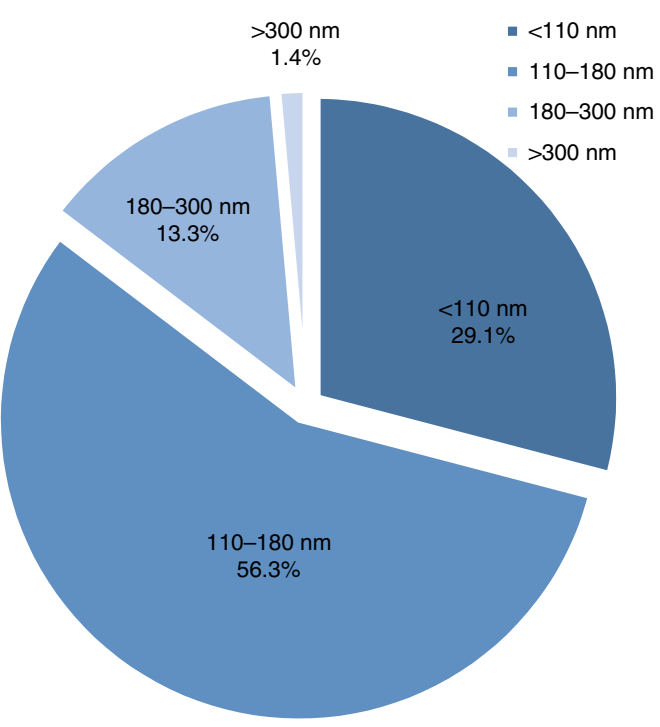

c

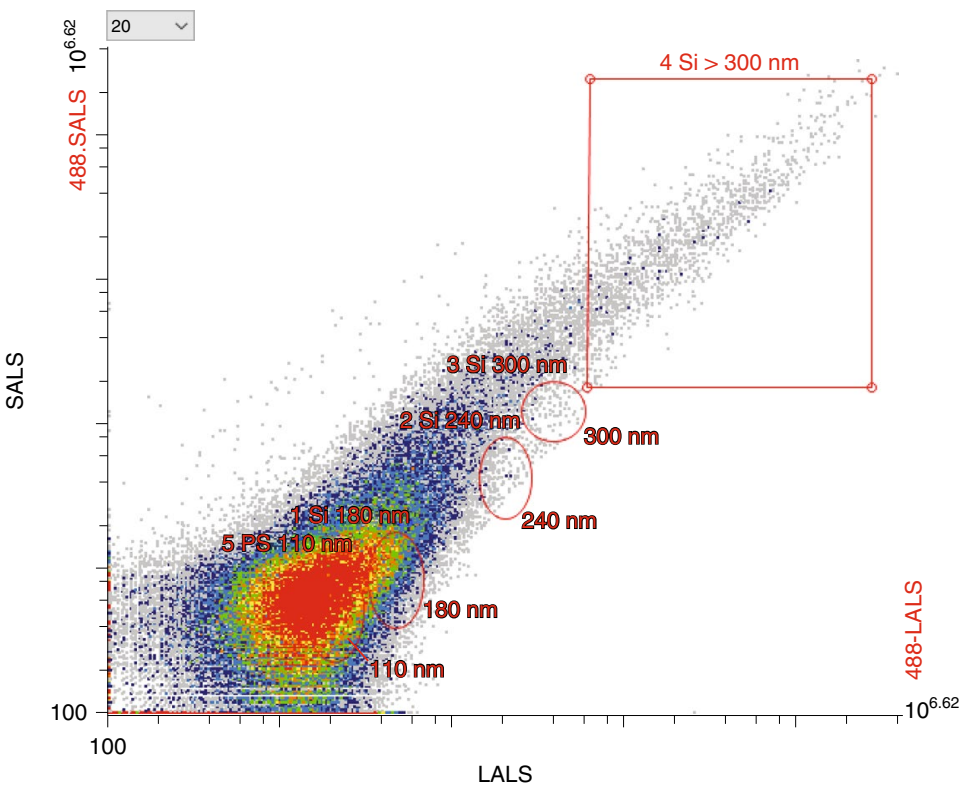



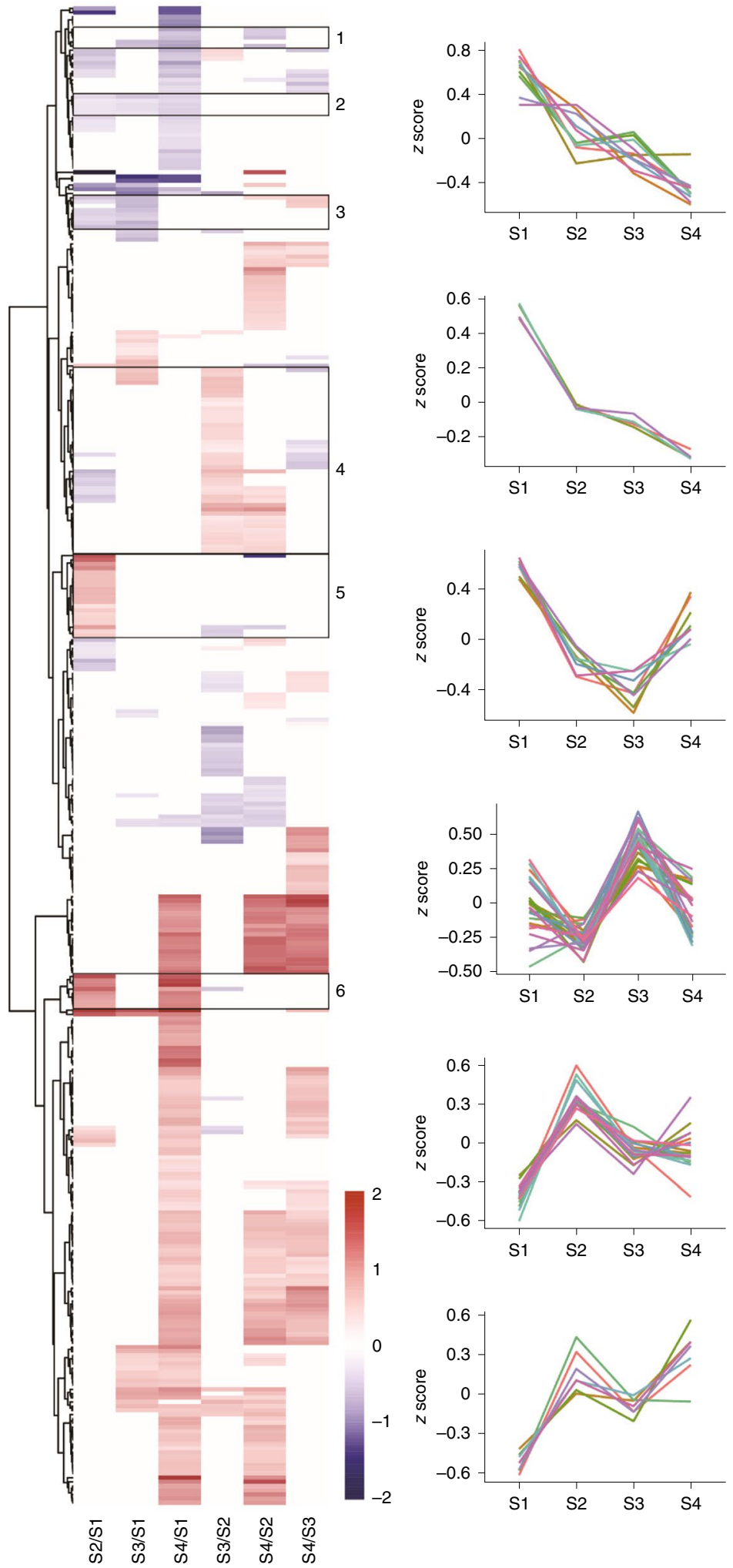

Fig. 2 Distinct temporal patterns of change in the lipidome of EVs in COVID-19. Hierarchical clustering was performed to aggregate lipids that exhibited similar patterns of change along the progressive course of COVID-19. $n=16,25,19$ and 18 independent patient samples for S1, S2, S3 and S4 stages, respectively. Clustering patterns were visually examined and six clusters ((1)-(6)) of EV membrane lipids were expanded for further discussion. Left, for the global heat map, $\log _{2}$-transformed fold changes were plotted. Middle, for the line plots, $z$ scores of mean of lipid levels expressed in MFM were plotted individually for lipids within each of the six clusters. Right, zoomed-in images of the six lipid clusters boxed in the global heat map. Statistical comparisons across temporal stages of COVID-19 were conducted using two-sided Kruskal-Wallis rank-sum tests and two-sided Dunn's tests were used for pairwise comparisons. Only fold changes with $P<0.05$ for each pairwise comparison are illustrated (that is, coloured blue or red); red indicates increases and blue indicates decreases in the respective phases analysed (for example, phase 2 relative to phase 1(S2/S1)). All fold changes with $P>0.05$ were set to 1 (shown in white). Gb3, globotriaosylceramide; PS, phosphatidylserine; SL, sulfatide; BMP, bis(monoacylglycero)phosphate; Cer, ceramide; LacCer, lactosylceramide.
(1) S4-specific reduction

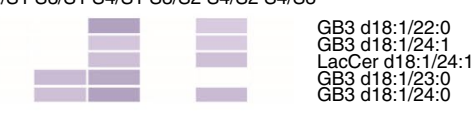

(2) S1-specific increase

$\mathrm{S} 2 / \mathrm{S} 1 \mathrm{~S} 3 / \mathrm{S} 1 \mathrm{~S} 4 / \mathrm{S} 1 \mathrm{~S} 3 / \mathrm{S} 2 \mathrm{~S} 4 / \mathrm{S} 2 \mathrm{~S} 4 / \mathrm{S} 3$

E40:6p (18:0/22:6)

(3) Reduction in S2/S3

$\mathrm{S} 2 / \mathrm{S} 1 \mathrm{~S} 3 / \mathrm{S} 1 \mathrm{~S} 4 / \mathrm{S} 1 \mathrm{~S} 3 / \mathrm{S} 2 \mathrm{~S} 4 / \mathrm{S} 2 \mathrm{~S} 4 / \mathrm{S} 3$

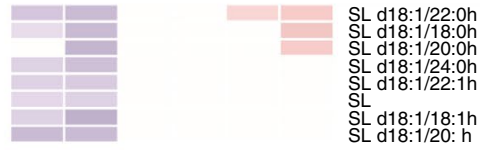

(4) S3-specific increase

$\mathrm{S} 2 / \mathrm{S} 1 \mathrm{~S} 3 / \mathrm{S} 1 \mathrm{~S} 4 / \mathrm{S} 1 \mathrm{~S} 3 / \mathrm{S} 2 \mathrm{~S} 4 / \mathrm{S} 2 \mathrm{~S} 4 / \mathrm{S} 3$

(5) S2-specific increase

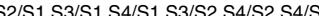

(6) increase in S2 and S4

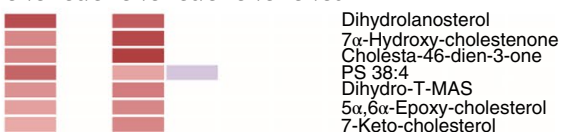

PE38:6p $(16: 0 / 22: 6)$

S2/S1 S3/S1 S4/S1 S3/S2 S4/S2 S4/S3 
a conventional indicator of cholesterol biosynthesis in the circulation $^{40}$, was distinctly increased in S4 (Fig. 3b), which suggests active cholesterol biosynthesis. Sitosterol, a phytosterol that exhibits moderate effects against viral replication ${ }^{41}$ and is an indicator of intestinal cholesterol absorption ${ }^{40}$, was also elevated in S4. Thus, the convalescent S4 stage had an EV sterol profile that is indicative of active cholesterol biosynthesis and uptake.

In addition to sterols, we noted appreciable increases in triacylglycerols (TAGs) in the convalescent S4 stage (Fig. 3c). Chord diagrams illustrating the lipid correlations in each temporal stage revealed a particularly strong positive correlation between EV TAGs and major membrane lipid classes (Fig. 3d), including PC, phosphatidylethanolamine (PE), PEp, phosphatidylglycerol (PG) and phosphatidylinositol (PI), in S1. Such correlations were lost in S2, and were progressively resumed in S3 and S4. The positive coregulation between TAGs and membrane phospholipids in S4, however, was still weaker compared to S1, which was evident in the reduced thickness of connecting blue lines between these lipid classes. These correlations suggest that the mobilization of storage TAGs that drives membrane phospholipid biogenesis was intense in S1, abrogated in S2, and then partially restored in S3 and S4. The ratio of storage TAGs to their lipolytic substrates, including diacylglycerols (DAGs) and free fatty acids (FFAs) (that is, TAG/(DAG + FFA)), was significantly highest in S4 (Extended Data Fig. 3b), indicating suppressed lipolysis in the convalescent phase. In our subsequent EV treatment of A549 cells, both Gene Ontology: Biological Process (GO:BP) 'GO:BP positive regulation of triglyceride lipase activity' and Reactome 'glycerophosholipid biosynthesis' pathways were elevated in cells treated with S1 EVs compared with S4 EVs (Supplementary Table 2).

Principal component analysis of EV proteomes comprising 142 identified proteins showed that the S1 and S2 stages were well segregated (Fig. 4a,b). Kyoto Encyclopedia of Genes and Genomes (KEGG) pathway enrichment analyses using differentially expressed proteins (DEPs) showed complement and coagulation cascades and platelet activation as the top dysregulated pathways during the course of COVID-19 (Fig. 4c). The complement system, as an integral component of the innate response that fights viruses primarily through the induction of proinflammatory responses ${ }^{42}$, was implicated in the pathogenesis of COVID-19 (ref. ${ }^{43}$ ). Pharmacological agents that impede the complement cascade have been proposed as potential therapeutics for COVID-19 (refs. ${ }^{44,45}$ ). We observed distinct increases in complement C1r and C1s (Fig. 4e), but not in complement C1q (Extended Data Fig. 4a,b), in S1 EVs. Complement C1q, C1r and C1s are subcomponents of the $\mathrm{C} 1$ complex that constitutes the classical pathway of complement activation $^{46}$. Localization of classic complement proteins in plasma EVs has been reported previously ${ }^{47}$. Complement $\mathrm{C} 8 \gamma$ chain, an essential component of the membrane attack complex ${ }^{46}$, was also specifically elevated in S1 EVs (Fig. 4e). By contrast, the EV proteome of S2 was marked by host-driven responses to curb viral spreading. Increase in integrin $\beta 3$ (Fig. 4e), which has been reported to suppress the intracellular entry of SARS-CoV-2 by blocking viral binding to the ACE2 receptor ${ }^{48}$, was observed in S2 EVs. SARS-CoV-2 has uniquely acquired an integrin-binding Arg-Gly-Asp (RGD) motif compared with other coronaviruses ${ }^{49}$, and circulating integrins can possibly bind to and occupy the receptor-binding domains of the spike protein of SARS-CoV-2 to sterically hinder virus-receptor binding ${ }^{48}$. S2-specific increase in the human leukocyte antigen class I histocompatibility antigen B- $\alpha$ chain (HLA-B) was also observed (Fig. 4e). HLA-B presents viral epitopes to cytolytic CD8 ${ }^{+} \mathrm{T}$ lymphocytes and triggers the elimination of infected cells ${ }^{50}$. S2 EVs also showed marked increases in proteins implicated in hypercoagulopathy, including fibrinogen- $\gamma$ chain, fibrinogen- $\alpha$ chain and coagulation factor $\mathrm{V}$, which are part of the innate immune response (GO:0045087) and interferon- $\gamma$-mediated signalling (GO:0060333) (Fig. 4d). Induction of T-cell-mediated defence and thrombus formation can constrain the continual spreading of SARS-CoV-2. EV-localized increases in fibrinogen- $\gamma$-chain proteins, which are specifically enriched in liver hepatocytes (The Human Protein Atlas), suggest that the liver may increase EV secretion to promote coagulopathy on a systems level (described further below).

The transition from the hyperinflammatory to resolution stage denotes a critical turning point of the disease course. We observed elevated proportion of raft lipids (that is, $(\mathrm{SM}+$ cholesterol)/PC) (Fig. 5a) and further validated the increased EV membrane anisotropy (Fig. 5b) in the hyperinflammatory compared with the resolution phase. The increases in raft lipids and EV lipid membrane anisotropy, coupled with a reduction in the steroidal GS modulator 3 3 -hydroxy-5-cholestenoic acid in S2, prompted us to look into the level of GS proteins localized in exosome-enriched EVs. We found that-in agreement with the endocytic localization of GS function ${ }^{51}$ - the catalytic subunit of GS (that is, PS-1) was enriched in exosome-enriched EVs relative to plasma (Fig. 5c) and PS-1 was specifically elevated in S2 compared with the other temporal stages (Fig. 5c,d). Retention of cholesterol in the endocytic compartments induces PS- 1 accumulation and increases the production of $A \beta 40$ and $A \beta 42$ (ref. ${ }^{52}$ ). Accordingly, we found that relative to S3, the level of A $\beta 40$ was significantly higher in S2 (Extended Data Fig. 5a). To determine the contribution of EVs from lung epithelial cells during COVID-19, we analysed the content of pulmonary surfactant-associated protein $\mathrm{C}$ (SP-C) in the isolated EVs. SP-C, which is exclusively expressed in lung tissues, is a predominantly hydrophobic protein that associates with lipids and is primarily synthesized in type II alveolar epithelial cells ${ }^{53}$. We found that mature SP-C (Fig. 5c,d) and its precursor protein (Extended Data Fig. 4c) were increased in EVs from S2, S3 and S4 stages compared with $\mathrm{S} 1$. EV release from the lungs therefore probably increases after the emergence of symptoms. S2 EVs also exhibited increased levels of interleukin-6 (IL-6) and cleaved caspase 3 (Fig. 5c,d and Extended Data Fig. 4d). IL-6 is a key player in the systemic hyperinflammation that is triggered by SARS-CoV-2 infection ${ }^{54}$. NOTCH-1 activation

Fig. 3 | Perturbed lipid homeostasis along the disease trajectory of COVID-19. a,b, The temporal changes in major lipid classes (a) and individual oxysterols and sterols (b) in plasma EVs from four phases of COVID-19. Statistical significance from two-sided Kruskal-Wallis rank-sum tests is indicated with an asterisk next to the lipid name; statistical significance from pairwise comparisons is indicated using a letter-based representation in which two groups sharing a common letter are not statistically different at $P<0.05$. c, The top lipids that were most significantly different in each pairwise comparison between two consecutive temporal stages defined based on both magnitudes of the $P$ value and fold changes (FC). a-c, Two-sided Dunn's tests were used for pairwise comparisons; $P<0.05$ was considered statistically significant. d, The lipid correlations between major EV lipid classes. Data were log-transformed and correlations between lipids were calculated using Spearman's correlations analysis for each stage of COVID-19. The width of the band indicates the number of significant correlations and the colour indicates the direction of correlation. The correlation coefficient cut-off was set to $\geq 0.7$ and $P$-value cut-off was set to $P<0.05$. Blue shade indicates positive correlations and red shade negative correlation between two connecting lipid classes. a-d, $n=16,25,19$ and 18 independent patient samples for S1, S2, S3 and S4 stages, respectively. LPC, Iysophosphatidylcholine; LPA, Iysophosphatidic acid; LPI, lysophosphatidylinositol; GM3, monosialodihexosyl ganglioside; PCp, plasmalogen phosphatidylcholine; GluCer, glucosylceramide; FF-MAS; follicular fluid meiosis-activating sterol; Sph, sphingosine; Odd-PC, phosphatidylcholine with odd-chain fatty acyls; PG, phosphatidylglycerol; S1P, sphingosine-1-phosphate. 
induces IL-6 production in a positive feedback loop that triggers hyperinflammatory responses ${ }^{16}$. Activated caspase 3 induces apoptosis and triggers the NLRP3 inflammasome to generate proinflammatory signals ${ }^{55}$.

We next treated A549 alveolar epithelial cells (as a cell type representing the primary infection site) and HepG2 cells (hepatocytes, representing a secondary, distal site of infection) with plasma EVs isolated from healthy control individuals and different temporal stages of COVID-19 (Fig. 5e-g). Hepatocytes were chosen for investigation because liver dysfunction-as indicated by abnormal levels of alanine aminotransferase and aspartate aminotransferase-was observed in $14-53 \%$ of patients with COVID-19 over the course of disease progression ${ }^{56}$. Visible uptake of EVs stained using the PKH67 dye into HepG2 cells was already observed at $4 \mathrm{~h}$ after a

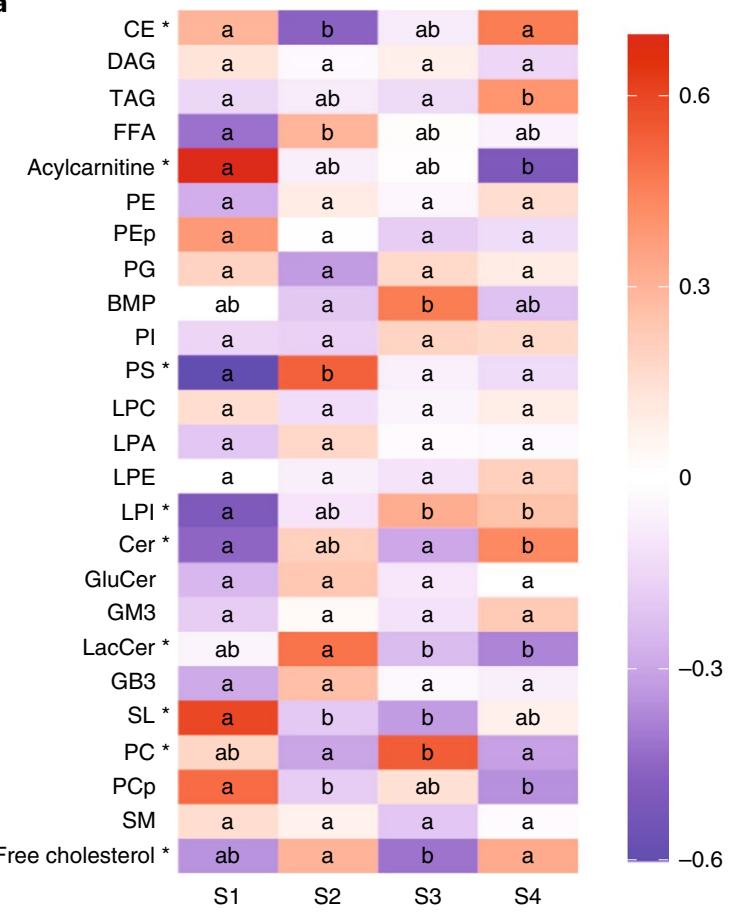

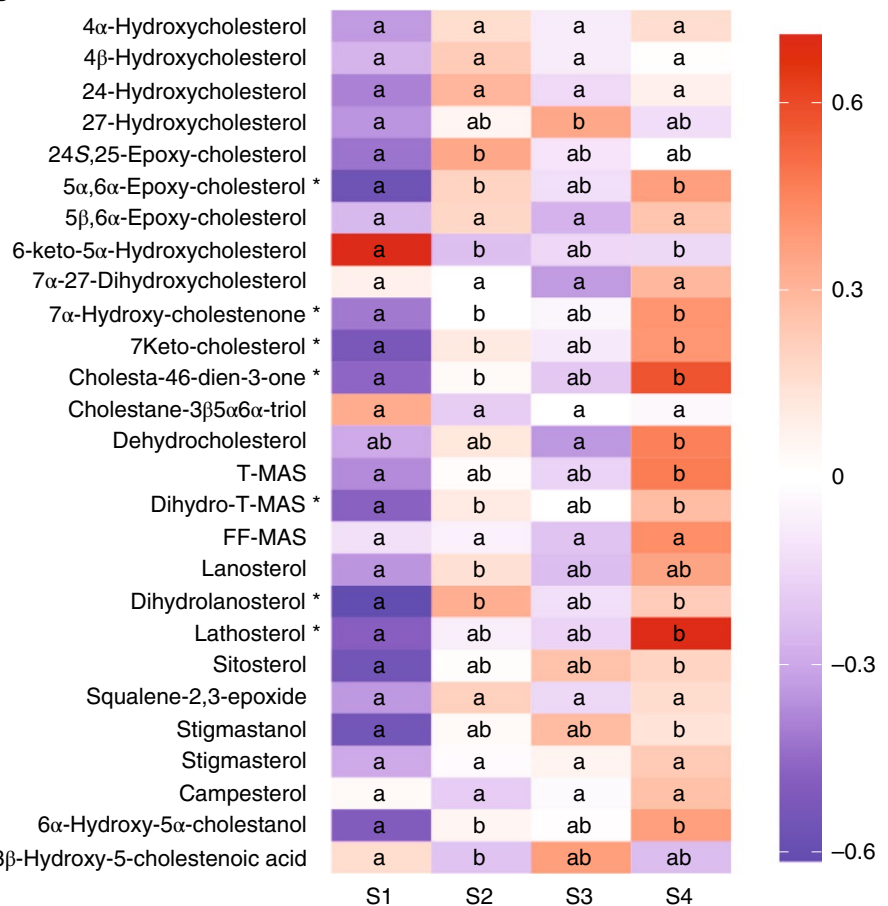

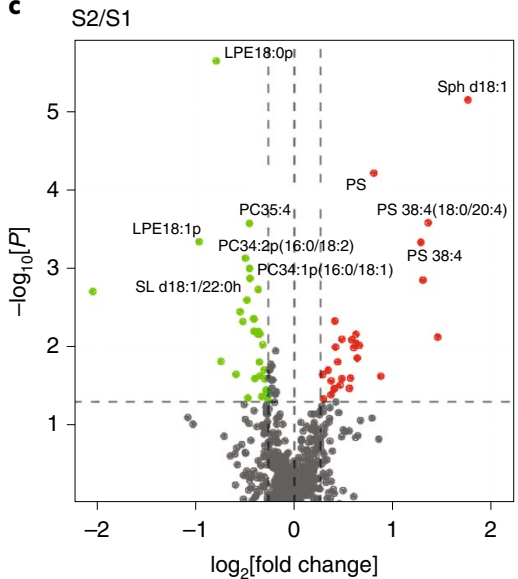

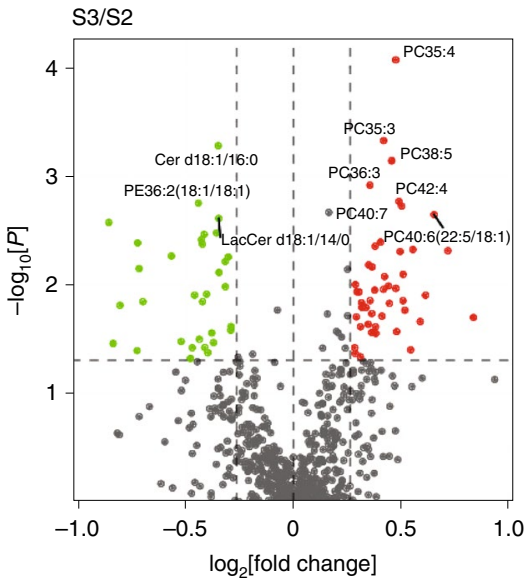
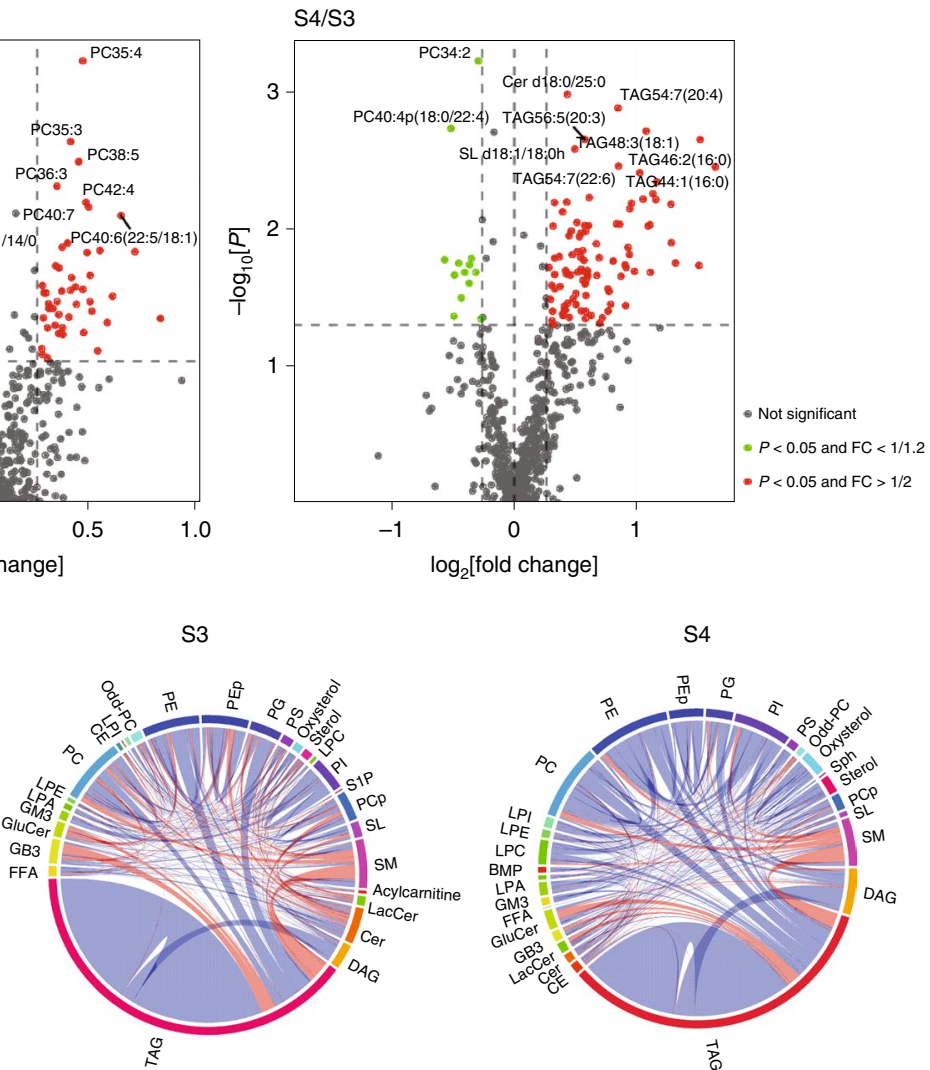


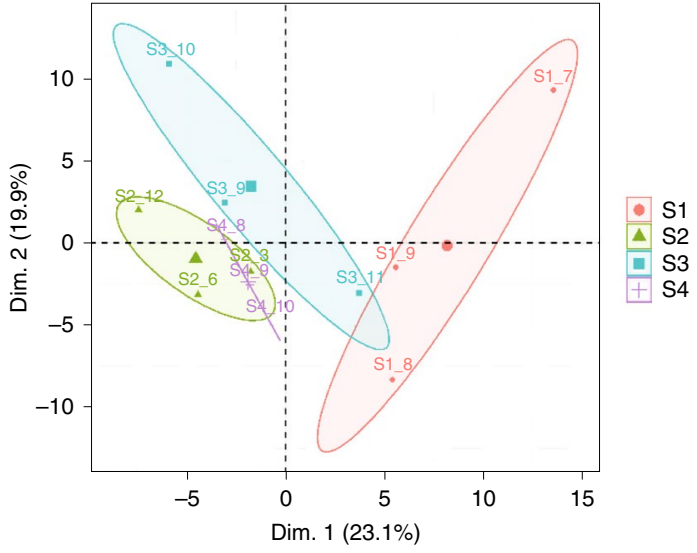

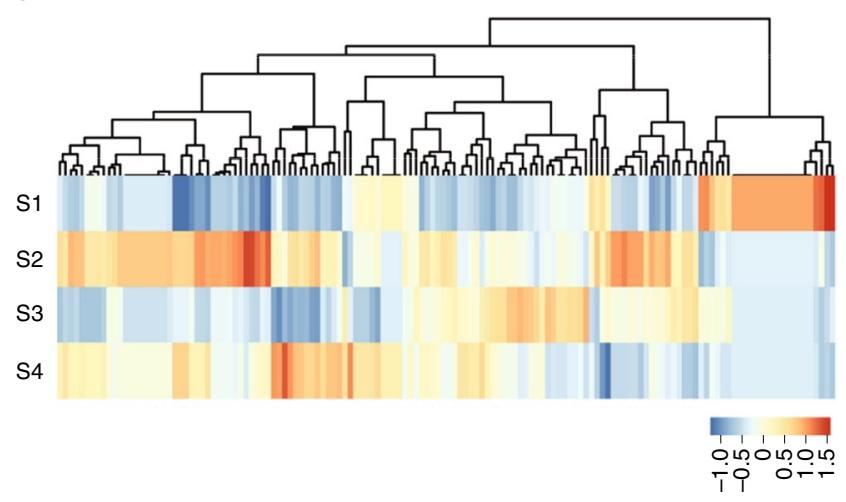

c

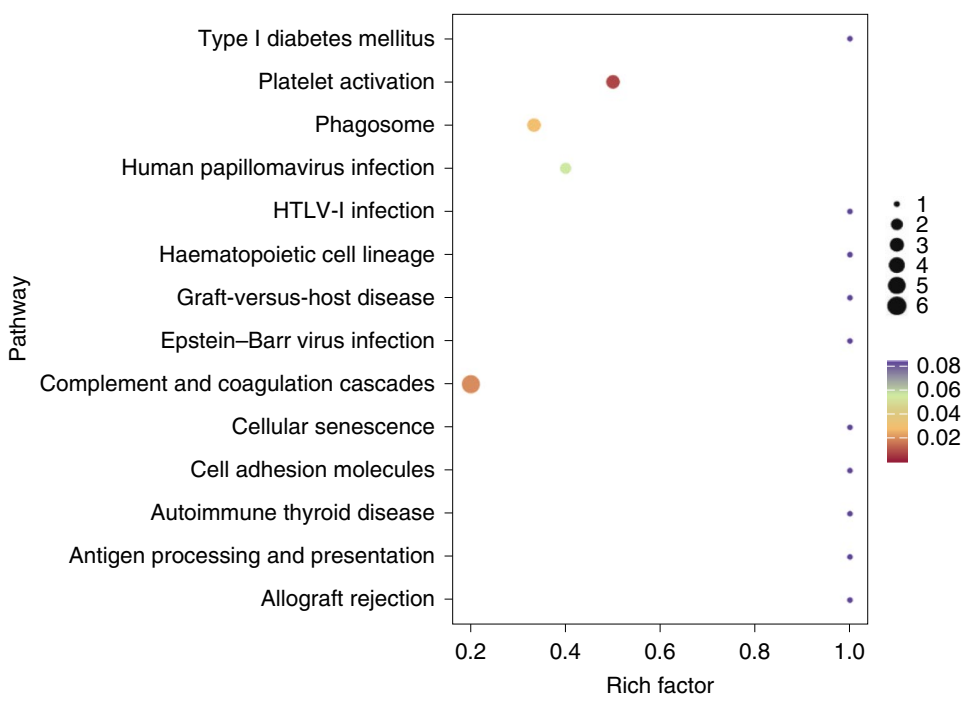

d

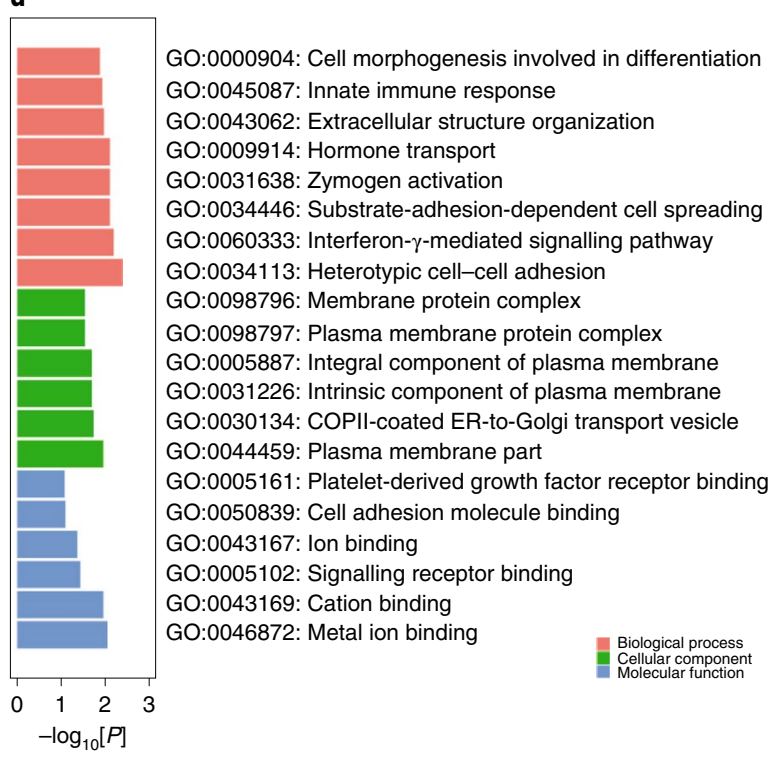

e

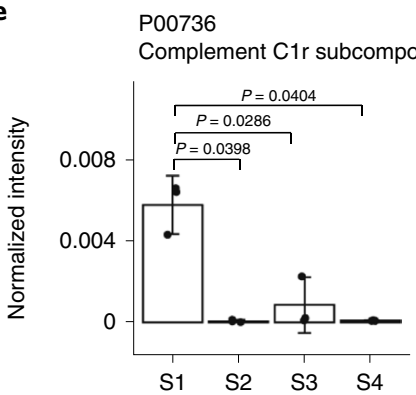

P01889 HLA-B

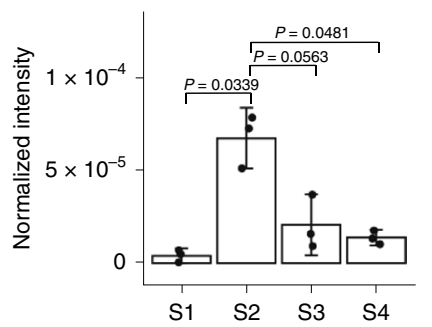

P09871

Complement $\mathrm{C} 1$ s subcomponent

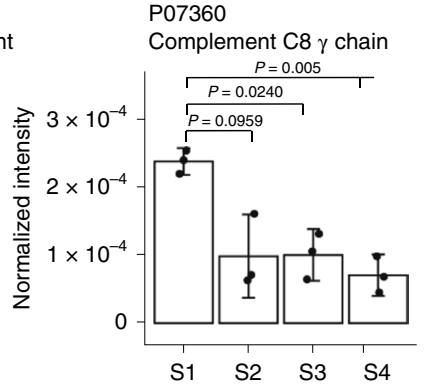

P02679

Fibrinogen- $\gamma$ chain
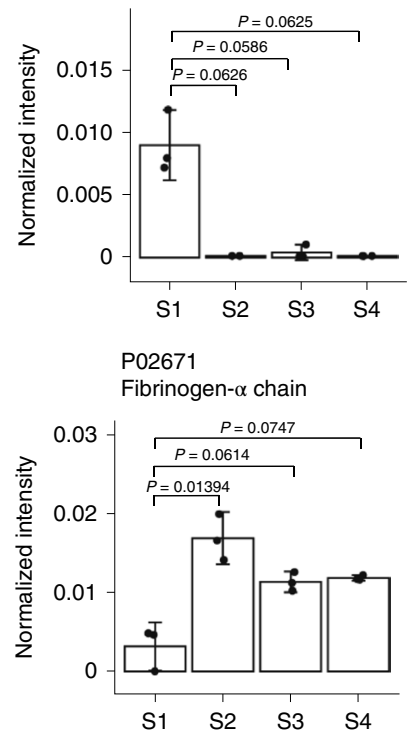

P02671

Fibrinogen- $\alpha$ chain

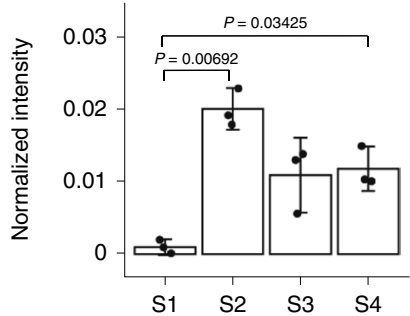

P05106

Integrin $\beta 3$

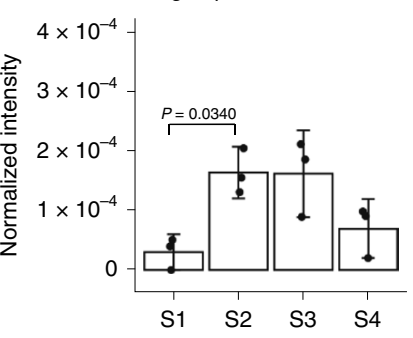

P12259

Coagulation factor $\mathrm{V}$

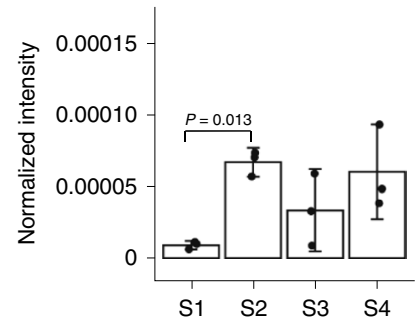

Fig. 4 | Temporal changes in EV proteome along four stages of COVID-19. a, The segregation of the EV proteome analysed by principal component analysis. Bracketed numbers indicate the percentage of total variance explained by each component. Dim., dimension. b, A library of 142 identified proteins from plasma EVs. c, Top KEGG pathways based on pathway enrichment analysis of DEPs. $\mathbf{d}$, Top terms from GO functional enrichment analyses based on DEPs. e, Changes in selected DEPs along the top dysregulated KEGG pathways. c-e, DEPs were defined on the basis of statistical significance ( $P<0.05)$ using two-sided GamesHowell's tests. $n=3$ independently pooled patient samples for each clinical phase. ER, endoplasmic reticulum; HTLV-1, human T-cell leukemia virus type 1. 
incubation at $37^{\circ} \mathrm{C}$ (Fig. 5e). To choose an optimized EV dosage for treatment, metabolic analysis using the Seahorse XFe96 extracellular flux analyser was conducted to measure the oxygen consumption rate of A549 and HepG2 cells that were incubated with increasing concentrations of EV proteins (Extended Data Fig. 6). The optimal dosage was determined by the minimum EV protein concentration that triggered significant reductions in the oxygen consumption rate and spare respiratory capacity in each cell type, which indicate changes in cell fitness and viability (which was $3.2 \mu \mathrm{g}$ per $2 \times 10^{4}$ A 549 cells and $6.4 \mu$ g per $2 \times 10^{4}$ HepG 2 cells).

We focused on pathways that were differentially regulated (that is, from upregulated to downregulated or vice versa) by EVs isolated from successive phases of COVID-19 (Fig. 5f,g). In A549 cells, the eukaryotic translation initiation factor kinase 4 (EIF2AK4) pathway was suppressed by S2 EVs, but upregulated by S3 and S4 EVs (Fig. 5f). Response of EIF2AK4 (GCN2) to amino acid deficiency was also among the top altered pathways after SARS-CoV-2 infection of A549 cells $^{57}$. Recessive mutations in EIF2AK4 are associated with pulmonary veno-occlusive disease ${ }^{58}$. Pathways governing eukaryotic translation, such as Reactome 'eukaryotic translation initiation' and KEGG 'ribosome' pathways, were suppressed by S2 EVs, but upregulated by EVs from the resolution and convalescent stages (Fig. 5f,g). SARS-CoV-2 interferes with host protein translation and protein trafficking to suppress the defences of the host against infection ${ }^{59}$, which is mediated by the viral non-structural protein 1 (NSP1 $^{60}$. Indeed, the Reactome 'translation of SARS-CoV-1 structural proteins' pathway was upregulated in A549 cells treated with S2 EVs compared with S3 EVs (Supplementary Table 2). As we did not detect SARS-CoV-2 NSP1 proteins in both immunoblot (Supplementary Figure 1) and proteomic database searches against the deposited NSP1 sequence, we think that these effects may be mediated by EV viral RNA cargoes that were not characterized in the present study. A distinct set of differentially regulated pathways emerged in EV-treated HepG2 cells (Fig. 5f,g). The Reactome 'pre-Notch expression and processing' pathway was increased in HepG2 cells treated with S2 EVs. Quantitative reverse-transcription PCR (RT-qPCR) analyses of HES1 and HES5 (Extended Data Fig. 5b), the downstream transcriptional targets of Notch activation in the liver $^{61,62}$, also support that Notch signalling was increased in HepG2 cells treated with EVs from the hyperinflammatory compared with the resolution phase. The Reactome 'haemostasis' pathway was upregulated by S2 EVs and downregulated by S3 EVs. The increased haemostasis triggered by S2 EVs in HepG2 cells agrees with our lipidomic and proteomic observations that these EVs were enriched in lipids and proteins that promote hypercoagulopathy.

Previous studies have proposed that host lipid metabolism is a primary target of positive single-strand RNA viruses such as SARS-CoV-2, which hijack the metabolism of the host to estab- lish a favourable micromilieu for productive infection ${ }^{63}$. Using high-coverage lipidomics and sterolomics, we discovered that the temporal trajectory of COVID-19 is marked by dysregulated cholesterol metabolism. In particular, cholesterol and oxysterol were reduced in the presymptomatic S1 phase, and were markedly increased in the hyperinflammatory S2 stage together with the manifestation of clinical symptoms. Cholesterol homeostasis and the retromer complex that regulates endosomal trafficking were among the key host pathways mediating SARS-CoV-2 infection of human cells $s^{13,14}$. A combinatorial approach of lipidomics and proteomics for the characterization of exosome-enriched EVs uncovered several notable compositional attributes of EVs, which may either reflect disease-stage-specific metabolism of the parent cells from which they originate, or confer distinct biological functions that render concerted metabolic response on a systems scale. In this study, we found that increases in raft lipids and membrane anisotropy of EVs during the hyperinflammatory S2 stage were associated with enhanced localization and activity of GS in these EVs. GS-mediated NOTCH-1 cleavage may activate the host inflammatory response to fight SARS-CoV-2 (ref. ${ }^{16}$ ). The reduction in raft lipids and PS-1 localization in EVs during the resolution phase may facilitate the cessation of Notch signalling and attenuate systemic inflammation (Fig. 5h). Increased $\mathrm{A} \beta$ peptide production indicates a possibly detrimental metabolic consequence of enhanced GS activity. The role of $A \beta$ peptides in triggering cognitive impairment has been extensively reported ${ }^{64}$. Neuropsychiatric deficits such as cognitive impairment and brain fog are well-recognized aspects of post-acute COVID-19 syndrome ${ }^{65}$. Nevertheless, A $\beta$ peptides also possess antiviral and antimicrobial properties ${ }^{6,67}$, for example, through the induction of the innate immune response mediated by the NALP3 inflammasome ${ }^{68}$. With regard to the liver, activation of Notch signalling in hepatocytes was previously shown to induce liver fibrosis in nonalcoholic fatty liver disease ${ }^{62}$. Our RNA-sequencing (RNA-seq) analyses indicated that in contrast to S3 EVs, S2 EVs impose metabolic stress on HepG2 cells. The KEGG 'oxidative phosphorylation' pathway was downregulated in HepG2 cells treated with S2 EVs (Fig. 5g). Compared to S2, the pathways 'WP resistin as a regulator of inflammation' and KEGG 'type II diabetes mellitus' were both downregulated when cells were treated with S3 EVs (Supplementary Table 2). Resistin is an intrahepatic cytokine expressed in response to injury and proinflammatory stimuli ${ }^{69}$, and hepatic resistin was reported to predict the severity of liver fibrosis in humans $\mathrm{s}^{70}$.

In summary, we present a temporal interrogation of the compositional changes in the lipidome and proteome of exosome-enriched EVs, and provide omics-based evidence of the underlying metabolic consequences during the clinical trajectory of COVID-19. Changing EV lipid membrane fluidity can alter the localization of protein cargoes, with EVs from different stages of COVID-19

Fig. 5 | Lipid membrane anisotropy alters the composition and function of EVs. a, Changes in the proportion of raft lipids ((SM + cholesterol)/PC) across temporal stages of COVID-19. $n=16,25,19$ and 18 independent patient samples for S1, S2, S3 and S4 stages, respectively. $P$ values from two-sided Dunn's tests are indicated. Statistical significance is indicated using a letter-based representation in which two groups sharing a common letter are not statistically different at $P<0.05$. b. Membrane anisotropy $(\gamma)$ in S2 and S3 EVs measured by incorporation of the fluorescent dye 1,6-diphenyl-1,3,5-hexatriene.

$n=4$ independent experiments. $P$ value from a two-sided Wilcoxon rank-sum test is indicated. $\mathbf{c}$, Immunoblot analysis of the PS-1 C-terminal fragment (PS-1(CTF)) - the catalytic subunit of GS-indicates that PS-1 is enriched in exosomal fractions (EXO) relative to the supernatant (SUP) and plasma. Immunoblot analysis of PS-1(CTF), precursor pulmonary surfactant protein-C (pre-SP-C), mature SP-C, IL-6, caspase-3 and cleaved caspase 3 (activated) in EVs across four stages of COVID-19. Equal amounts of total proteins (30 $\mu$ g) were loaded in each lane. $\mathbf{d}$, Quantified levels of PS-1(CTF), SP-C, IL-6 and cleaved caspase 3 for the four temporal stages. $P$ values from two-sided Dunn's tests are shown. c,d, $n=3,4,3$ and 3 independent patient samples for S1, S2, S3 and S4 stages, respectively. e, Confocal images of the uptake of PKH67-stained EVs (green) into cultured HepG2 cells after $4 \mathrm{~h}$ of incubation at $37^{\circ} \mathrm{C}$ compared with the negative control in which PBS was added. Cell nuclei were stained with DAPI (blue). Representative images are from three independent experiments. Scale bars, $20 \mu \mathrm{m} . \mathbf{f}, \mathbf{g}$, Top 20 pathways from the Reactome pathway database (f) and KEGG pathway database (g) based on nominally significantly enriched gene sets obtained using gene set enrichment analysis (GSEA). $n=3$ independent cultures for each treatment condition. h, Transition from S2 hyperinflammatory to S3 resolution phase is marked by a proportional reduction in EV membrane raft lipids (SM + cholesterol). The resultant decrease in membrane anisotropy alters the localization of PS-1 and other protein cargoes, providing EVs from distinct stages of infection with different biological properties. ECM, extracellular matrix; HC, healthy control individual; HDACs, histone deacetylases; elFs, eukaryotic initiation factors. 
having distinct biological attributes. In particular, we found that exosome-enriched EVs from the resolution phase trigger opposing effects on cellular metabolism in vitro, and thus may be able

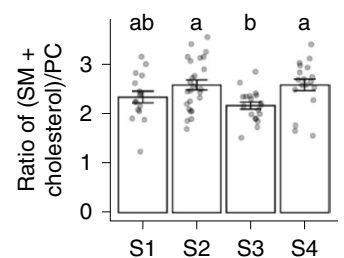

d
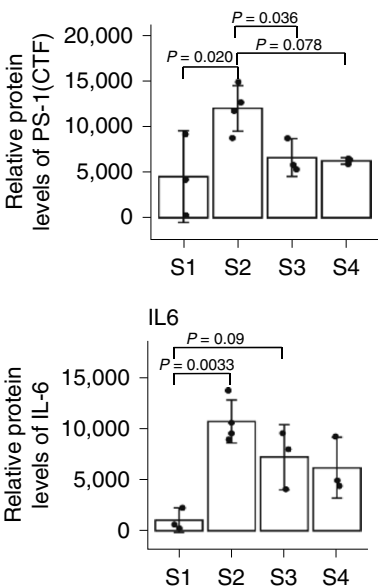

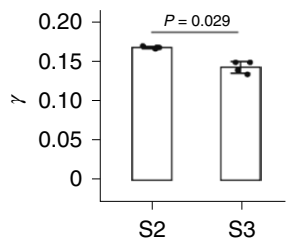

to offset cellular responses mounted by S2 EVs. Although different proteins and RNA cargoes not characterized in this study may also contribute to the defined EV functions, we showed that enhanced

PS-1(CTF)

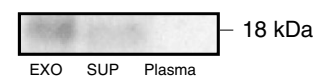

PS-1(CTF)

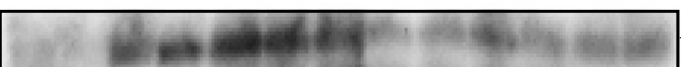

$(\mathrm{kDa})$

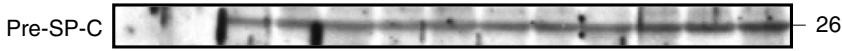
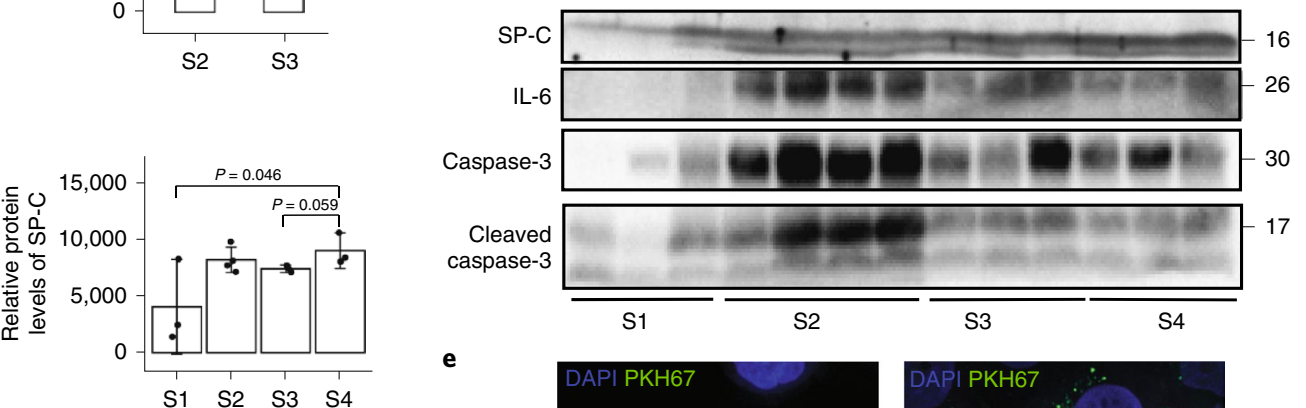

Cleaved
caspase-3

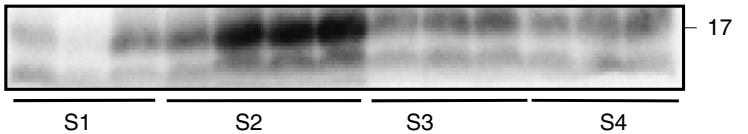

e

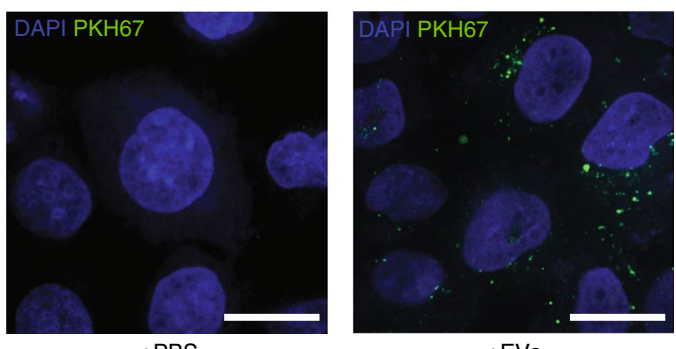

+ PBS

$+\mathrm{EVs}$
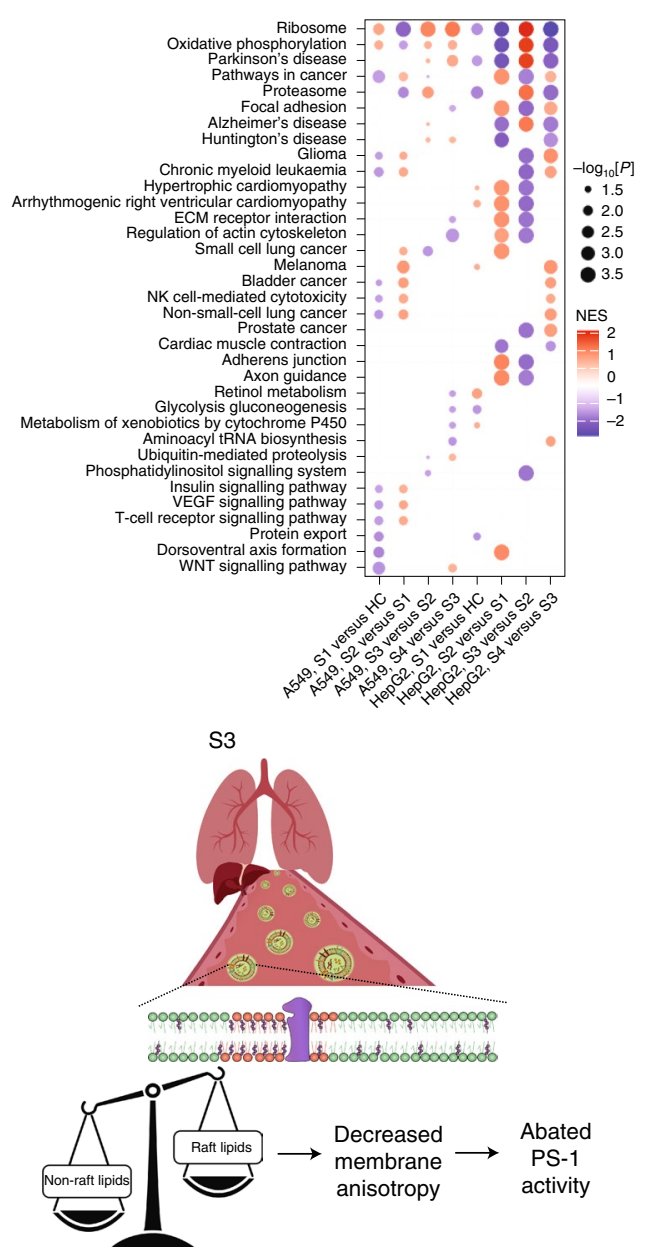
PS-1 localization to exosome-enriched EVs in the hyperinflammatory S2 phase compared to the resolution S3 phase contributes to their distinct cellular responses on recipient cells. These data also serve as a useful repository of information for future research to elucidate the mechanistic clues that underlie the metabolic dysregulation and consequences in COVID-19 pathogenesis.

\section{Methods}

Study design and participants. This retrospective cohort study included two cohorts of inpatients from the Fifth Medical Center of Chinese PLA General Hospital and the Beijing Ditan Hospital Capital Medical University, which were designated hospitals for patients with COVID-19 in Beijing, China. Patients were diagnosed according to the WHO interim guidance and only survivors of COVID-19 were included in this study. We investigated four stages (S1-S4) across the clinical course of SARS-CoV-2 infection (Fig. 1a). Patient blood samples were assigned to four different stages based on clinical records of SARS-CoV-2 RNA results and laboratory findings on the date of blood collection, as well as hospital admission and discharge dates relative to the blood sampling date (Supplementary Table 1).S1 refers to presymptomatic individuals with positive results for SARS-CoV-2 RNA but who do not yet to exhibit any associated symptoms (that is, dry cough, fever and so on). S1 represents the incubation phase of COVID-19 with active SARS-CoV-2 replication and blunted innate immune response $^{22}$. S2 comprises symptomatic patient samples collected within the first week of symptom onset with positive SARS-CoV-2 RNA results, a period for which the viral load of patients with COVID-19 is expected to peak followed closely by hyperinflammation ${ }^{71,72}$. S3 consists of post-hyperinflammation samples with negative SARS-CoV-2 results. S4 refers to convalescent patient samples collected within $3 \mathrm{~d}$ or on the day of hospital discharge, when clinical symptoms were reasonably resolved. Baseline characteristics and laboratory findings of patients with COVID-19 were summarized in Table 1 . The clinical data were collected and checked independently by two physicians. This study was approved by the ethics committee of the Fifth Medical Center of the PLA General Hospital and written informed consent was obtained from all participants enrolled in this study.

Plasma collection and EV isolation. All blood samples were collected after an overnight fast in BD Vacutainer (BD 367525). Plasma was separated by centrifugation at $733 \mathrm{~g}$ for $10 \mathrm{~min}$. EVs were isolated from plasma using the Total Exosome Isolation Kit from Plasma (Thermo Fisher Scientific) according to the manufacturer's instructions, with slight modifications. Plasma $(200 \mu \mathrm{l})$ was first centrifuged at $2,000 \mathrm{~g}$ for $20 \mathrm{~min}$ to remove cells and debris. The clear supernatant was transferred to a fresh tube, and centrifuged again at $10,000 \mathrm{~g}$ for $20 \mathrm{~min}$ to further remove finer debris and large microvesicles. The resultant supernatant was then diluted with $100 \mu \mathrm{l}$ of ice-cold $1 \times$ PBS and mixed thoroughly by vortexing. To minimize artificial oxidation, $2 \mu \mathrm{g}$ of butylated hydroxytoluene (BHT) was immediately added to the diluted plasma, which was then incubated at $37^{\circ} \mathrm{C}$ for $10 \mathrm{~min}$ with $10 \mu \mathrm{l}$ of proteinase $\mathrm{K}$ to eliminate non-exosomal plasma proteins. At the end of the proteinase K treatment, $60 \mu \mathrm{l}$ of Exosome Precipitation Reagent was added and the samples were incubated at $4{ }^{\circ} \mathrm{C}$ for $30 \mathrm{~min}$ for EV precipitation. After the incubation, the samples were centrifuged at $10,000 \mathrm{~g}$ for $5 \mathrm{~min}$. Isolated EVs were collected as a pellet, while the supernatant comprising plasma contents without EVs were transferred to fresh tube and stored at $-80^{\circ} \mathrm{C}$. Isolated EVs were resuspended in $100 \mu \mathrm{l}$ of ice-cold $1 \times \mathrm{PBS}$, and immediately inactivated in $650 \mu \mathrm{l}$ ice-cold chloroform:methanol $(1: 2, \mathrm{v} / \mathrm{v})$ containing $1 \%$ BHT $(\mathrm{w} / \mathrm{v})$ for oxysterols and lipid extraction (see the next section).

Lipid extraction from EVs. Lipids were extracted following a modified Bligh and Dyer's protocol as previously described ${ }^{10}$. EVs were incubated in extraction solvent comprising chloroform:methanol $(1: 2, \mathrm{v} / \mathrm{v})$ containing $1 \% \mathrm{BHT}$ at $4{ }^{\circ} \mathrm{C}$ for $1 \mathrm{~h}$ at 1,500 r.p.m. After the incubation, $350 \mu$ ice-cold Milli-Q water and $250 \mu \mathrm{l}$ ice-cold chloroform were added to induce phase separation. The samples were centrifuged at $16,260 \mathrm{~g}$ at $4{ }^{\circ} \mathrm{C}$ for $5 \mathrm{~min}$. The lower organic phase containing lipids and oxysterols was transferred to a new tube. The extraction was repeated once through the addition of another $450 \mu \mathrm{l}$ ice-cold chloroform to the remaining aqueous phase, and the extractions were pooled and dried in a SpeedVac under organic mode. The lipid extract was resuspended in $200 \mu \mathrm{l}$ chloroform:methanol $(1: 1, \mathrm{v} / \mathrm{v})$. One-tenth of the lipid extract from EVs was used for targeted lipidomics analysis, and ninth-tenth of the extract was dried and used for oxysterol extraction and derivatization (see the next section). The upper aqueous-methanol phase together with the remnant EV pellets was dried in a SpeedVac under $\mathrm{H}_{2} \mathrm{O}$ mode, and the dried pellets were channelled for protein extraction and analyses.

Oxysterol extraction and derivatization. Free oxysterols were analysed in this study. In brief, the lipid extract was resuspended in $500 \mu \mathrm{l}$ of ethanol containing $5 \mu \mathrm{g}$ BHT. An internal standard cocktail ( $50 \mu \mathrm{l})$ comprising $\mathrm{d}_{7}$ 24-hydroxycholesterol, $\mathrm{d}_{7}-7 \beta$-hydroxycholesterol, $\mathrm{d}_{6}-25$-hydroxycholesterol, $\mathrm{d}_{7}$ 27-hydroxycholesterol, $\mathrm{d}_{7}-7 \alpha, 24$-dihydroxycholestenone, $\mathrm{d}_{7}-7$-keto-cholesterol, $\mathrm{d}_{7}-7 \alpha$-hydroxycholestenone, $\mathrm{d}_{6}$-lanosterol, $\mathrm{d}_{7}$-lathosterol, $\mathrm{d}_{6}$-T-MAS, $\mathrm{d}_{7}$ -

7 -dehydrocholesterol and $\mathrm{d}_{6}-\beta$-sitosterol (Avanti Polar Lipids) was added to the samples. The samples were incubated at 1,200 r.p.m. for $15 \mathrm{~min}$ at $4{ }^{\circ} \mathrm{C}$. At the end of the incubation, $250 \mu \mathrm{l}$ of Milli-Q water and $1 \mathrm{ml}$ of $n$-hexane was added. The samples were mixed thoroughly by vortexing, then centrifuged at $16,260 \mathrm{~g}$ for $5 \mathrm{~min} 4^{\circ} \mathrm{C}$. The clear upper phase containing the oxysterols and sterols in hexane was transferred to new tube. The extraction was repeated once with another $1 \mathrm{ml}$ of $n$-hexane. The pooled extract was dried in a SpeedVac under organic mode. Oxysterols and sterols were derivatized to obtain their picolinic acid esters before HPLC-MS/MS analysis as previously described ${ }^{73,74}$.

Lipidomics and sterolomics analysis. The EV lipidome was quantified using a high-coverage targeted lipidomic approach as described previously ${ }^{10}$, but with further refinement in the selection of internal standards used for quantification. Analyses of phospholipids and sphingolipids were conducted on a system comprising an Exion-UPLC coupled with a 6500 Plus QTRAP that runs Analyst v.1.6.3 (Sciex); neutral lipid analyses were carried out on an Agilent 1260 HPLC (Agilent Technologies) connected to a 5500 QTRAP that runs Analyst v.1.6.3 (Sciex). The internal standard cocktail was adjusted according to the endogenous abundances of various lipid classes in isolated EVs, which comprised $\mathrm{d}_{9}$-PC32:0(16:0/16:0), $\mathrm{d}_{9}$-PC36:1p(18:0p/18:1), $\mathrm{d}_{7}$-PE33:1(15:0/18:1), $\mathrm{d}_{9}$-PE36:1p(18:0p/18:1), $\mathrm{d}_{31}$-phosphatidylserine (16:0/18:1), $\mathrm{d}_{7}$-PG33:1(15:0/18:1), $\mathrm{d}_{7}$-PI33:1(15:0/18:1), d PA33:1(15:0/18:1), C14-BMP, $\mathrm{d}_{8}$-SM d18:1/18:1, Cer d18:1/d $\mathrm{d}_{7}-15: 0$, GluCer d18:1/8:0, $\mathrm{d}_{3}$-LacCer d18:1/16:0, GM3 d18:1/18:0- $\mathrm{d}_{3}$, Gb3-d18:1/17:0, sulfatide-d18:1/17:0, $\mathrm{d}_{7}$-LPC 18:1, $\mathrm{d}_{7}$-LPE 18:1, LPA-C17:0,

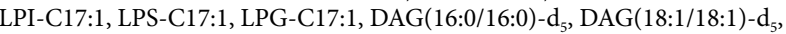
$\mathrm{d}_{7}$-cholesteryl sulfate, S1P-d17:1 and Sph-d17:1 obtained from Avanti Polar Lipids, TAG(14:0) $)_{3}-\mathrm{d}_{5}$, TAG $(16: 0)_{3}-\mathrm{d}_{5}$, TAG(18:0) ${ }_{3}-\mathrm{d}_{5}, \mathrm{~d}_{6}-\mathrm{CE} 18: 0$ and $\mathrm{d}_{6}$-cholesterol purchased from CDN Isotopes, and $\mathrm{d}_{3}-16: 0$-carnitine from Cambridge Isotope Laboratories. $d_{31}$-FFA-16:0 from Sigma-Aldrich and $d_{8}$-FFA-20:4 from Cayman Chemicals were used for quantification of saturated/monounsaturated fatty acids and polyunsaturated fatty acids, respectively. Oxysterols and sterols were separated on a $1.9 \mu \mathrm{m}$ Hypersil Gold C18-selectivity column $(50 \times 2.1 \mathrm{~mm})$ (Thermo Fisher Scientific) and analysed on an U3000-DGLC coupled with a QTRAP 6500 Plus (Sciex) in their derivatized forms, as described previously ${ }^{74}$. A gradient system comprising acetonitrile:methanol:water (45:45:40, v/v/v) containing $0.1 \%$ acetic acid as mobile phase $\mathrm{A}$ and acetonitrile:methanol:water $(45: 45: 10, \mathrm{v} / \mathrm{v} / \mathrm{v})$ containing $0.1 \%$ acetic acid as mobile phase B was used at a flow rate of $550 \mu \mathrm{min}^{-1}$ and column oven temperature at $35^{\circ} \mathrm{C}$. The gradient program adopted was as follows: $0-2 \mathrm{~min}, 5 \% \mathrm{~B}$; $2-3 \mathrm{~min}, 5-25 \% \mathrm{~B}$; 3-6 min, $25-65 \% \mathrm{~B}$; $6-8 \mathrm{~min}, 65-100 \% \mathrm{~B} ; 8-12 \mathrm{~min}, 100 \% \mathrm{~B} ; 12-13 \mathrm{~min}, 100-5 \% \mathrm{~B} ; 13-15 \mathrm{~min}: 5 \% \mathrm{~B}$. The identities of individual oxysterols were validated based on both the multiple reaction monitoring transitions and retention time relative to their authentic reference compounds. Quality-control samples were inserted every ten samples throughout the batch analysis, and the performance of quality-control samples is illustrated in Supplementary Figs. 2, 3. PC, phosphatidylcholine; PCp, plasmalogen phosphatidylcholine; PE, phosphatidylethanolamine; PEp, plasmalogen phosphatidylethanolamine; PG, phosphatidylglycerol; PI, phosphatidylinositol; PA, phosphatidic acid; BMP, bis(monoacylglycero)phosphate; SM, sphingomyelin; Cer, ceramide; GluCer, glucosylceramide; LacCer, lactosylceramide; GM3, monosialodihexosyl ganglioside; Gb3, globotriaosylceramide; LPC;lysophosphatidylcholine; LPE, lysophosphatidylethanolamine; LPA, lysophosphatidic acid; LPI, lysophosphatidylinositol; LPS, lysophosphatidylserine; LPG, lysophosphatidylglycerol; DAG, diacylglycerol; S1P, sphingosine1-phosphate; Sph, sphingosine; TAG, triacylglycerol; CE, cholesteryl ester; FFA, free fatty acid.

Proteomic analysis. Approximately $150 \mu \mathrm{g}$ of EV protein was obtained from each sample after lipid and oxysterol extraction. Proteomic analyses were conducted using three pooled samples from each temporal stage. Each sample was pooled from two randomly selected samples in the same clinical group. Each pooled sample $\left(50 \mu \mathrm{g}\right.$ protein) was reconstituted using $8 \mathrm{M}$ urea in $50 \mathrm{mM} \mathrm{NH}_{4} \mathrm{HCO}_{3}$. The proteins were then digested with trypsin as previously described ${ }^{75}$. In brief, the proteins were reduced with $10 \mathrm{mM} \mathrm{DTT}$ at $37^{\circ} \mathrm{C}$ for $1 \mathrm{~h}$ and then alkylated with $25 \mathrm{mM}$ iodoacetamide at room temperature for $1 \mathrm{~h}$ in the dark. After reduction and alkylation, the lysates were diluted with $6 \times$ the volume of $50 \mathrm{mM} \mathrm{NH}_{4} \mathrm{HCO}_{3}$, and digested with trypsin at $37^{\circ} \mathrm{C}$ for $18 \mathrm{~h}$ using an enzyme:substrate ratio of 1:50. All tryptic peptides were desalted by StageTip and dried with a SpeedVac before use. The resuspended peptides were analysed on a LTQ Orbitrap Elite mass spectrometer running Xcalibur v.4.1 (Thermo Fisher Scientific) coupled online to an Easy-nLC 1000 (Thermo Fisher Scientific) in a data-dependent mode. The peptides were separated by reverse-phase LC with a $150 \mu \mathrm{m}$ (inner diameter) $\times$ $250 \mathrm{~mm}$ (length) analytical column packed with C18 particles of $1.9 \mu \mathrm{m}$ diameter. The mobile phases comprise buffer A $\left(100 \% \mathrm{H}_{2} \mathrm{O}, 0.1 \%\right.$ formic acid) and buffer $\mathrm{B}$ ( $100 \%$ acetonitrile, $0.1 \%$ formic acid), and a 180 -min non-linear gradient was used for chromatographic separation. All MS measurements were performed in positive ion mode. Precursor ions were measured in the Orbitrap analyser at 240,000 resolution and a target value of $10^{6}$ ions was used for each MS scan. The twenty most intense ions from each MS scan were isolated, fragmented and measured in the linear ion trap. The collision-induced dissociation normalized collision energy was set to 35 . The data were analysed using a pre-release version of Thermo 
Scientific Proteome Discoverer software v.2.3. The Homo sapiens proteome sequence database containing 20,417 entries downloaded from UniProt was used for the database searching. Normalization was performed against the total peptide intensity of each sample.

Nanoscale flow cytometry analysis. EVs were analysed using an A50 Micro Nanoscale Flow Cytometer (Apogee FlowSystems) equipped with a blue $(488 \mathrm{~nm}$ ) laser $^{76}$. Before analysis, all samples were diluted tenfold to avoid a swarm effect. Parameters in the control panel were set as follows, sheath pressure $=150 \mathrm{mbar}$, number of flush cycles $=2$. Calibration of flow cytometer performance is conducted using a standard reference bead mix (ApogeeMix, Apogee Flow Systems) composed of a mixture of silica nanoparticles with diameters of $180 \mathrm{~nm}$, $240 \mathrm{~nm}, 300 \mathrm{~nm}, 590 \mathrm{~nm}, 880 \mathrm{~nm}$ and $1,300 \mathrm{~nm}$, and green fluorescent polystyrene nanoparticles of diameters $110 \mathrm{~nm}$ and $500 \mathrm{~nm}$. The threshold of medium angle light scatter (ALS) is set at 22 arbitrary units to eliminate optical and electronic background noise without losing particles of interest. Photomultiplier tube voltages were set to $324 \mathrm{~V}$ for small ALS, $350 \mathrm{~V}$ for medium ALS and $291 \mathrm{~V}$ for large ALS for size analysis. All measurements were performed in log mode. Noise levels in the photomultiplier tube panel were kept below 0.5 . All data were analysed using the Apogee Histogram Software.

Transmission electron microscopy. Transmission electron microscopy images of EVs were taken on the basis of a previous protocol ${ }^{77}$. In brief, isolated EVs were reconstituted in $200 \mu \mathrm{l}$ of $1 \times$ PBS and diluted $10 \times$ and $500 \times$. EV samples at two different dilutions were mixed with an equal volume of $4 \%$ paraformaldehyde. Drops of fixed EVs $(5 \mu \mathrm{l})$ were deposited on clean parafilm, and a formvar-carbon-coated grid was floated on each drop of fixed EVs with the coated side facing down. The samples were left to dry at room temperature for $20 \mathrm{~min}$ and then washed with PBS. Next, samples were fixed in $1 \%$ glutaraldehyde for $5 \mathrm{~min}$, washed with deionized water eight times, and stained with $3 \%$ uranyl acetate for $5 \mathrm{~min}$. Excess liquid was removed and the stained EVs were observed using a transmission electron microscope (JEM 1400) at $80 \mathrm{kV}$.

EV membrane fluidity assay. EV membrane fluidity was measured using the fluorescent dye 1,6-diphenyl-1,3,5-hexatriene (Sigma-Aldrich, D208000) as previously described ${ }^{78}$. In brief, 10-nmol-equivalent PCs from EVs, quantified on the LC-MS using $\mathrm{d}_{9}$-PC32:0(16:0/16:0) as an internal standard, were resuspended in $1 \mathrm{ml}$ of HEPES buffer containing protease inhibitors at $\mathrm{pH}$. The optical density of the mixture was checked to be $<0.08$ at $348 \mathrm{~nm}$ to ensure subsequent fluorescent measurements were not optically obscured. Labelling of EV membranes with 1,6-diphenyl-1,3,5-hexatriene probes was carried out at a molecular ratio of probe:PC of 1:360 for $30 \mathrm{~min}$ at room temperature with gentle agitation in the dark. The labelled samples were excited at $35^{\circ} \mathrm{C}$ (to mimic body temperature) with vertically polarized light in a Hitachi F-7000 fluorescence spectrophotometer. Emission intensities were measured parallel (IVV) and perpendicular (IVH) to the vertical plane of polarization. To eliminate the effect of instrument polarization response, a grating correction factor $(G)$ was taken into consideration, calculated by exciting the samples with horizontally polarized light and measuring the parallel and perpendicular components, where $G=\mathrm{IHV} / \mathrm{IHH}$. Anisotropy $(\gamma)$ was calculated according to the equation (1),

$$
\gamma=(\mathrm{IVV}-\mathrm{GIVH}) /(\mathrm{IVV}+2 \mathrm{GIVH})
$$

Four replicates of EV samples isolated from pooled plasma of S2 and S3 states were measured.

Cell culture. HepG2 and A549 cells were obtained from the American Type Culture Collection. HepG2 cells were maintained in DMEM (high-glucose) medium (Gibco) supplemented with $10 \%$ FBS, and A549 were maintained in RPMI 1640 medium (Gibco) supplemented with 10\% FBS. Cells were cultured at $37^{\circ} \mathrm{C}$ in an atmosphere of $5 \% \mathrm{CO}_{2}$.

Confocal imaging of EV uptake by HepG2 cells. EVs isolated from the plasma of patients with COVID-19 were stained with PHK67 dye (Sigma-Aldrich, MINI67) according to the manufacturer's instructions. In brief, $100 \mu \mathrm{l}$ of resuspended PKH67 was added to either $100 \mu \mathrm{LV}$ suspension or PBS (negative control). EVs were evenly mixed with the dye solution and left to stand at room temperature for $5 \mathrm{~min}$. Next, $200 \mu \mathrm{l}$ of $1 \%$ bovine serum albumin was added to bind excess dye, and the samples were transferred to $100-\mathrm{kDa}$ Vivaspin filters and centrifuged at $16,260 \mathrm{~g}$ for $3 \mathrm{~min}$. The upper phase containing the stained EVs was washed twice with $1 \times$ PBS, and incubated with HepG2 cells for $4 \mathrm{~h}$ at $37^{\circ} \mathrm{C}$. At the end of the incubation, the cells were stained with DAPI and observed using a confocal laser scanning microscope (Observer Z1, Carl Zeiss)

Treatment of HepG2 and A549 cells with EVs. EVs were isolated from $1 \mathrm{ml}$ of pooled plasma from each of the five clinical stages, which included healthy control individuals, as well as individuals from the S1, S2, S3 and S4 phases of COVID19. Cells $\left(10^{6}\right.$ per well) were incubated with culture medium containing the appropriate concentrations (measured in terms of quantity of EV proteins) of EVs (that is, $160 \mu \mathrm{g}$ per well for A549 cells; $320 \mu \mathrm{g}$ per well for HepG2 cells) in 12-well plates for $12 \mathrm{~h}$ at $37^{\circ} \mathrm{C}$. At the end of the incubation, the cell culture medium was aspirated. The cells were washed three times with ice-cold $1 \times$ PBS. Cells designated for transcriptome analyses were immediately inactivated through the addition of TRIzol reagent (Invitrogen, 15596-026). Three separate wells for each clinical group were collected for transcriptome analyses.

RNA analysis using RT-qPCR. Total RNA was extracted from cells inactivated with $1 \mathrm{ml}$ of TRIzol reagent (Invitrogen, 15596-026). For each phase separation, $0.2 \mathrm{ml}$ of chloroform was added and the aqueous phase was collected in fresh tubes and mixed with $0.5 \mathrm{ml}$ of isopropyl alcohol to pellet the RNA. RNA pellets were washed with $75 \%$ ethanol and digested with DNase for $30 \mathrm{~min}$ to remove DNA contamination, then pelleted through the addition of $\mathrm{NaOAc}$ and $\mathrm{LiCl}$. RNA pellets were then washed with $75 \%$ ethanol and reconstituted in nuclease-free water. cDNA was synthesized using the iScript cDNA Synthesis kit (Bio-Rad, 1708891). RT-qPCR was performed using the SYBR Green PCR kit (Bio-Rad, 1725120) on a Bio-Rad CFX Connect 96-Real-Time PCR Detection machine. Relative expression values of HES1 and HES5 were normalized to those of GAPDH. Gene-specific primers used for amplification are listed in Supplementary Table 3.

PromethION library preparation and sequencing. First-strand cDNA was synthesized in a $20 \mu \mathrm{l}$ reaction mixture with $13 \mu \mathrm{l}$ of purified nucleic acids from each sample and $100 \mathrm{pmol}$ of oligo(dT)15 primers. For the double-stranded cDNA synthesis, $100 \mathrm{pmol}$ of random primers (Promega, C118A) was added. PromethION library preparation was performed according to the manufacturer's instructions for barcoding cDNA (SQK-LSK109, EXP-NBD104 and EXP-NBD114). When multiplexing, all of the samples were pooled together. ONT MinKNOW software (v.19.10.1) was used to collect raw sequencing data, and Guppy (v.3.2.4) was used for real-time base calling. The PromethION was run for up to $48 \mathrm{~h}$. Adaptor and low-quality sequences of raw data were trimmed using Qcat with the default parameters. Trimmed reads were aligned to the human genome (version GRCH38) using mimimap2 (ref. ${ }^{79}$ ). The number of reads aligned to human genes was calculated using BEDtools multicov with the human gene annotation file GFF (GENCODE v.37).

Western blot analysis. Proteins were extracted using RIPA lysis buffer with protease inhibitor cocktail (Sigma-Aldrich). The protein content was determined using a Pierce BCA protein assay kit (Thermo Fisher Scientific) according to the manufacturer's instructions. After boiling, lysates containing $30 \mu \mathrm{g}$ protein were subjected to SDS-PAGE and blotted onto nitrocellulose membranes (Pall). Primary antibodies included: anti-CD9 (Santa Cruz, sc-13118, 1:500), anti-CD63 (Santa Cruz, sc-5275, 1:500), anti-TSG101 (Abcam, ab125011, 1:2,500), anti-PS-1 (Abcam, ab76083, 1:2,000), anti-caspase-3 (CST, 9662s, 1:2,000), anti-SP-C (Santa-Cruz, sc-13979, 1:500), anti-IL-6 (Abcam, ab233706, 1:2,000), anti-annexin A1 (CST, 32934, 1:1,000), anti-ApoB (Merck Millipore, AB742, 1:3000) and anti-ApoE (Merck Millipore, AB947, 1:1,000) antibodies. Secondary antibodies included: goat anti-rabbit HRP (ZSGB-BIO, ZB-2305, 1:5,000), goat anti-mouse HRP (ZSGB-BIO, ZB-2301, 1:5,000) and rabbit anti-goat HRP (Biosharp, BL004A, $1: 2,500)$ antibodies.

Statistical analysis. To examine the changes in EV membrane lipid composition, for all statistical analyses, lipids and sterols were expressed in molar fractions normalized to total membrane lipids (that is, MFM), which comprises the summed levels of all phospholipids, sphingolipids and free cholesterol quantified in $\mu \mathrm{mol}$. Statistical comparisons between different temporal stages of COVID-19 for lipids were conducted using Kruskal-Wallis rank-sum tests and Dunn's tests for pairwise comparisons. $P<0.05$ was considered to be statistically significant. Statistical significance of all pairwise comparisons was indicated using a letter-based representation, in which two groups that share a common letter are not statistically different at the $P<0.05$ level. Fold change was calculated as the ratio of means of two groups. Hierarchical clustering using the Ward method (ward.D2 in R hclust) was performed on $\log _{2}$-transformed fold-change values, which were set to 1 (no change) if corresponding to $P>0.05$. Heat maps were drawn using ComplexHeatmap v.2.7.10.9001. Clustering was visually evaluated and patterns of interests were manually selected. For lipid species within each cluster, standardized group means (mean $=0$ and s.d. $=1$ ) of each group are presented as line plots. Standardized group means of lipid class sums and sterols are presented as a heat map, where $P<0.05$ in a Kruskal-Wallis test is indicated with an asterisk after the names, and pairwise comparison results from Dunn's tests are indicated in each cell using the letter-based representation. Fold change and $-\log _{10}[P]$ values are presented as volcano plots. Spearman correlation coefficients were calculated between lipid species within samples in each phase using Hmisc v.4.4-2. Correlations with corresponding $P<0.05$ were visualized using chord diagrams drawn with chorddiag v.0.1.2 and circlize v.0.4.12, in which the band width indicates the number of correlations and the colour indicates the direction of correlation. The correlation coefficient cut-off was set to $\geq 0.7$ and the $P$-value cut-off was set to $P<0.05$. The blue shade indicates positive correlations and the red shade indicates negative correlation between lipids. Statistical analyses were performed using R v.4.0.2. 
For analysis of proteomic data, proteins with missing intensity values in more than one sample were removed and not analysed. Remaining missing values were replaced by zeroes. The intensities of individual proteins were normalized to the sum of total intensities in each sample before statistical analyses. Statistical significance for pairwise comparisons between temporal stages was assessed using Games-Howell's test. Pathway analyses were conducted using DEPs. DEPs were assigned to GO local database annotations in Perl scripts using Fisher's exact tests, for which proteins were categorized into three groups, namely biological process, cellular component and molecular function. Pathway enrichment analysis was carried out using the KEGG database (http://www.genome.jp/kegg/pathway.html) using Fisher's exact tests in Perl scripts.

For analysis of RNA-seq data, differentially expressed genes between the various clinical groups were obtained using the $\mathrm{R}$ package DESeq2 (see source data associated with Fig. 5). The Wald statistics from each of the comparisons were then used for pre-ranked GSEA analysis (10,000 permutations) using the fgsea package in R v.3.6.2, ClusterProfiler v.3.14.3 and Bioconductor v.3.1. Gene symbols were mapped to Entrez IDs using org.hs.eg.db v.3.10.0. Gene sets originating from KEGG, Reactome, GO and WikiPathways were obtained from MsigDB v.7.3 and used in the analysis. Summary plots of the nominally significantly enriched gene sets were plotted using ggplot2.

Reporting Summary. Further information on research design is available in the Nature Research Reporting Summary linked to this article.

\section{Data availability}

Proteomic data of exosome-enriched EVs have been deposited in the ProteomeXchange Consortium via the PRIDE partner repository under accession code PXD026091. RNA-seq data of EV-treated cells have been deposited in the GEO omnibus under accession code GSE174668. Minimal datasets including lipidomic, proteomic and RNA-seq data are provided as source data files. Other data supporting the findings of this study are available from the corresponding author upon reasonable request. Source data are provided with this paper.

Received: 6 November 2020; Accepted: 9 June 2021;

Published online: 22 June 2021

\section{References}

1. Mathieu, M., Martin-Jaular, L., Lavieu, G. \& Théry, C. Specificities of secretion and uptake of exosomes and other extracellular vesicles for cell-to-cell communication. Nat. Cell Biol. 21, 9-17 (2019).

2. Hendrix, A. The nature of blood(y) extracellular vesicles. Nat. Rev. Mol. Cell Biol. 22, 243 (2021).

3. Cocozza, F., Grisard, E., Martin-Jaular, L., Mathieu, M. \& Théry, C. SnapShot: extracellular vesicles. Cell 182, 262-262.e1 (2020).

4. Ayres, J. S. A metabolic handbook for the COVID-19 pandemic. Nat. Metab. 2, 572-585 (2020).

5. Harding, C., Heuser, J. \& Stahl, P. Receptor-mediated endocytosis of transferrin and recycling of the transferrin receptor in rat reticulocytes. J. Cell Biol. 97, 329-339 (1983).

6. Izquierdo-Useros, N. et al. HIV and mature dendritic cells: Trojan exosomes riding the Trojan horse? PLoS Pathog. 6, e1000740 (2010).

7. Cosset, F. L. \& Dreux, M. HCV transmission by hepatic exosomes establishes a productive infection. J. Hepatol. 60, 674-675 (2014).

8. Singh, P. P., LeMaire, C., Tan, J. C., Zeng, E. \& Schorey, J. S. Exosomes released from $M$. tuberculosis infected cells can suppress IFN- $\gamma$ mediated activation of naïve macrophages. PLoS ONE 6, e18564 (2011).

9. Subra, C., Laulagnier, K., Perret, B. \& Record, M. Exosome lipidomics unravels lipid sorting at the level of multivesicular bodies. Biochimie $\mathbf{8 9}$, 205-212 (2007).

10. Song, J. W. et al. Omics-driven systems interrogation of metabolic dysregulation in COVID-19 pathogenesis. Cell Metab. 32, 188-202.e185 (2020)

11. Möbius, W. et al. Recycling compartments and the internal vesicles of multivesicular bodies harbor most of the cholesterol found in the endocytic pathway. Traffic 4, 222-231 (2003).

12. Pfrieger, F. W. \& Vitale, N. Cholesterol and the journey of extracellular vesicles. J. Lipid Res. 59, 2255-2261 (2018).

13. Daniloski, Z. et al. Identification of required host factors for SARS-CoV-2 infection in human cells. Cell 184(92), 105.e16 (2021).

14. Hoffmann, H.-H. et al. Functional interrogation of a SARS-CoV-2 host protein interactome identifies unique and shared coronavirus host factors. Cell Host Microbe 29, 267-280.e5 (2021).

15. Beloribi, S. et al. Exosomal lipids impact notch signaling and induce death of human pancreatic tumoral SOJ-6 cells. PLoS ONE 7, e47480 (2012).

16. Rizzo, P. et al. COVID-19 in the heart and the lungs: could we "Notch" the inflammatory storm? Basic Res. Cardiol. 115, 31 (2020).

17. Aguayo-Ortiz, R., Straub, J. E. \& Dominguez, L. Influence of membrane lipid composition on the structure and activity of $\gamma$-secretase. Phys. Chem. Chem. Phys. 20, 27294-27304 (2018).
18. Holmes, O., Paturi, S., Ye, W., Wolfe, M. S. \& Selkoe, D. J. Effects of membrane lipids on the activity and processivity of purified $\gamma$-secretase. Biochemistry 51, 3565-3575 (2012).

19. Levi, M., Thachil, J., Iba, T. \& Levy, J. H. Coagulation abnormalities and thrombosis in patients with COVID-19. Lancet Haematol. 7, e438-e440 (2020).

20. Zhou, F. et al. Clinical course and risk factors for mortality of adult inpatients with COVID-19 in Wuhan, China: a retrospective cohort study. Lancet 395, 1054-1062 (2020).

21. McGonagle, D., O’Donnell, J. S., Sharif, K., Emery, P. \& Bridgewood, C. Immune mechanisms of pulmonary intravascular coagulopathy in COVID-19 pneumonia. Lancet Rheumatol. 2, e437-e445 (2020).

22. Acharya, D., Liu, G. \& Gack, M. U. Dysregulation of type I interferon responses in COVID-19. Nat. Rev. Immunol. 20, 397-398 (2020).

23. Muller, L., Hong, C. S., Stolz, D. B., Watkins, S. C. \& Whiteside, T. L. Isolation of biologically-active exosomes from human plasma. J. Immunol. Methods 411, 55-65 (2014).

24. Voisin, M. et al. Identification of a tumor-promoter cholesterol metabolite in human breast cancers acting through the glucocorticoid receptor. Proc. Natl Acad. Sci. USA 114, E9346-E9355 (2017).

25. Marcello, A. et al. The cholesterol metabolite 27-hydroxycholesterol inhibits SARS-CoV-2 and is markedly decreased in COVID-19 patients. Redox Biol. 36, $101682(2020)$

26. Puri, A. et al. The neutral glycosphingolipid globotriaosylceramide promotes fusion mediated by a CD4-dependent CXCR4-utilizing HIV type 1 envelope glycoprotein. Proc. Natl Acad. Sci. USA 95, 14435-14440 (1998).

27. Lorizate, M. et al. Comparative lipidomics analysis of HIV-1 particles and their producer cell membrane in different cell lines. Cell. Microbiol. 15, 292-304 (2013)

28. Honsho, M. \& Fujiki, Y. Plasmalogen homeostasis - regulation of plasmalogen biosynthesis and its physiological consequence in mammals. FEBS Lett. 591, 2720-2729 (2017).

29. Blomqvist, M. et al. Multiple tissue-specific isoforms of sulfatide activate CD1d-restricted type II NKT cells. Eur. J. Immunol. 39, 1726-1735 (2009).

30. Buschard, K. et al. Sulfatide controls insulin secretion by modulation of ATP-sensitive $\mathrm{K}^{+}$-channel activity and $\mathrm{Ca}^{2+}$-dependent exocytosis in rat pancreatic $\beta$-cells. Diabetes 51, 2514-2521 (2002).

31. Hollstein, T. et al. Autoantibody-negative insulin-dependent diabetes mellitus after SARS-CoV-2 infection: a case report. Nat. Metab. 2, 1021-1024 (2020).

32. Müller, J. A. et al. SARS-CoV-2 infects and replicates in cells of the human endocrine and exocrine pancreas. Nat. Metab. 3, 149-165 (2021).

33. Kapustin, A. N. \& Shanahan, C. M. Emerging roles for vascular smooth muscle cell exosomes in calcification and coagulation. J. Physiol. 594, 2905-2914 (2016).

34. Lembo, D., Cagno, V., Civra, A. \& Poli, G. Oxysterols: an emerging class of broad spectrum antiviral effectors. Mol. Asp. Med. 49, 23-30 (2016).

35. Segala, G. et al. Dendrogenin A drives LXR to trigger lethal autophagy in cancers. Nat. Commun. 8, 1903 (2017)

36. Küçük, O. et al. Inhibition of NK cell-mediated cytotoxicity by oxysterols. Cell. Immunol. 139, 541-549 (1992).

37. Küçük, O. et al. Inhibition of cytolytic T lymphocyte activity by oxysterols. Lipids 29, 657-660 (1994).

38. Jung, J. I. et al. Cholestenoic acid, an endogenous cholesterol metabolite, is a potent $\gamma$-secretase modulator. Mol. Neurodegener. 10, 29 (2015).

39. Jung, J. I. et al. Steroids as $\gamma$-secretase modulators. FASEB J. 27, 3775-3785 (2013).

40. Tilvis, R. S., Valvanne, J. N., Strandberg, T. E. \& Miettinen, T. A. Prognostic significance of serum cholesterol, lathosterol, and sitosterol in old age; a 17-year population study. Ann. Med. 43, 292-301 (2011).

41. Parvez, M. K. et al. Plant-derived antiviral drugs as novel hepatitis B virus inhibitors: cell culture and molecular docking study. Saudi Pharm. J. 27, 389-400 (2019).

42. Risitano, A. M. et al. Complement as a target in COVID-19? Nat. Rev. Immunol. 20, 343-344 (2020).

43. Shen, B. et al. Proteomic and metabolomic characterization of COVID-19 patient sera. Cell 182, 59-72.e15 (2020).

44. Stahel, P. F. \& Barnum, S. R. Complement inhibition in coronavirus disease (COVID)-19: a neglected therapeutic option. Front. Immunol. 11, 1661 (2020)

45. Thomson, T. M., Toscano-Guerra, E., Casis, E. \& Paciucci, R. C1 esterase inhibitor and the contact system in COVID-19. Br. J. Haematol. 190, 520-524 (2020).

46. Java, A. et al. The complement system in COVID-19: friend and foe? JCI Insight 5, e140711 (2020).

47. Looze, $\mathrm{C}$. et al. Proteomic profiling of human plasma exosomes identifies PPAR $\gamma$ as an exosome-associated protein. Biochem. Biophys. Res. Commun. 378, 433-438 (2009)

48. Luan, J., Lu, Y., Gao, S. \& Zhang, L. A potential inhibitory role for integrin in the receptor targeting of SARS-CoV-2. J. Infect. 81, 318-356 (2020). 
49. Sigrist, C. J., Bridge, A. \& Le Mercier, P. A potential role for integrins in host cell entry by SARS-CoV-2. Antivir. Res. 177, 104759 (2020).

50. La Porta, C. A. M. \& Zapperi, S. Estimating the binding of SARS-CoV-2 peptides to HLA class I in human subpopulations using artificial neural networks. Cell Syst. 11, 412-417.e412 (2020).

51. Choy, R. W., Cheng, Z. \& Schekman, R. Amyloid precursor protein (APP) traffics from the cell surface via endosomes for amyloid $\beta(\mathrm{A} \beta)$ production in the trans-Golgi network. Proc. Natl Acad. Sci. USA 109, E2077-E2082 (2012).

52. Runz, H. et al. Inhibition of intracellular cholesterol transport alters presenilin localization and amyloid precursor protein processing in neuronal cells. J. Neurosci. 22, 1679-1689 (2002)

53. Weaver, T. E. \& Whitsett, J. A. Function and regulation of expression of pulmonary surfactant-associated proteins. Biochem. J. 273, 249-264 (1991).

54. Zhang, C., Wu, Z., Li, J. W., Zhao, H. \& Wang, G. Q. Cytokine release syndrome in severe COVID-19: interleukin-6 receptor antagonist tocilizumab may be the key to reduce mortality. Int. J. Antimicrob. Agents 55, 105954 (2020)

55. Vince, J. E. et al. The mitochondrial apoptotic effectors BAX/BAK activate caspase- 3 and -7 to trigger NLRP3 inflammasome and caspase- 8 driven IL- $1 \beta$ activation. Cell Rep. 25, 2339-2353.e2334 (2018).

56. Zhang, C., Shi, L. \& Wang, F.-S. Liver injury in COVID-19: management and challenges. Lancet Gastroenterol. Hepatol. 5, 428-430 (2020).

57. Ibrahim, I. H. \& Ellakwa, D. E. SUMO pathway, blood coagulation and oxidative stress in SARS-CoV-2 infection. Biochem Biophys. Rep. 26, 100938 (2021).

58. Eyries, M. et al. EIF2AK4 mutations cause pulmonary veno-occlusive disease, a recessive form of pulmonary hypertension. Nat. Genet. 46, 65-69 (2014)

59. Banerjee, A. K. et al. SARS-CoV-2 disrupts splicing, translation, and protein trafficking to suppress host defenses. Cell 183, 1325-1339.e1321 (2020).

60. Schubert, K. et al. SARS-CoV-2 Nsp1 binds the ribosomal mRNA channel to inhibit translation. Nat. Struct. Mol. Biol. 27, 959-966 (2020).

61. Luiken, S. et al. NOTCH target gene HES5 mediates oncogenic and tumor suppressive functions in hepatocarcinogenesis. Oncogene 39, 3128-3144 (2020).

62. Zhu, C. et al. Hepatocyte Notch activation induces liver fibrosis in nonalcoholic steatohepatitis. Sci. Transl. Med. 10, eaat0344 (2018).

63. Pagliari, F. et al. ssRNA Virus and Host Lipid Rearrangements: Is There a Role for Lipid Droplets in SARS-CoV-2 Infection? Front. Mol. Biosci. 7, 578964 (2020)

64. Raven, F. et al. Soluble gamma-secretase modulators attenuate Alzheimer's $\beta$-amyloid pathology and induce conformational changes in presenilin 1 . EBioMedicine 24, 93-101 (2017).

65. Nalbandian, A. et al. Post-acute COVID-19 syndrome. Nat. Med. 27, 601-615 (2021).

66. Bourgade, K., Dupuis, G., Frost, E. H. \& Fülöp, T. Anti-viral properties of amyloid- $\beta$ peptides. J. Alzheimers Dis. 54, 859-878 (2016).

67. Soscia, S. J. et al. The Alzheimer's disease-associated amyloid $\beta$-protein is an antimicrobial peptide. PLoS ONE 5, e9505 (2010).

68. Halle, A. et al. The NALP3 inflammasome is involved in the innate immune response to amyloid- $\beta$. Nat. Immunol. 9, 857-865 (2008).

69. Bertolani, C. et al. Resistin as an intrahepatic cytokine: overexpression during chronic injury and induction of proinflammatory actions in hepatic stellate cells. Am. J. Pathol. 169, 2042-2053 (2006).

70. Shen, C. et al. The relationship between hepatic resistin overexpression and inflammation in patients with nonalcoholic steatohepatitis. $B M C$ Gastroenterol. 14, 39 (2014).

71. He, X. et al. Temporal dynamics in viral shedding and transmissibility of COVID-19. Nat. Med. 26, 672-675 (2020).

72. Zou, L. et al. SARS-CoV-2 viral load in upper respiratory specimens of infected patients. N. Engl. J. Med. 382, 1177-1179 (2020)
73. Honda, A. et al. Highly sensitive quantification of key regulatory oxysterols in biological samples by LC-ESI-MS/MS. J. Lipid Res. 50, 350-357 (2009).

74. Chen, L. et al. Endogenous sterol intermediates of the mevalonate pathway regulate HMGCR degradation and SREBP-2 processing. J. Lipid Res. 60, 1765-1775 (2019).

75. Washburn, M. P., Wolters, D. \& Yates, J. R.III Large-scale analysis of the yeast proteome by multidimensional protein identification technology. Nat. Biotechnol. 19, 242-247 (2001).

76. Cumba Garcia, L. M., Peterson, T. E., Cepeda, M. A., Johnson, A. J. \& Parney, I. F. Isolation and analysis of plasma-derived exosomes in patients with glioma. Front. Oncol. 9, 651 (2019).

77. Thery, C., Amigorena, S., Raposo, G. \& Clayton, A. Isolation and characterization of exosomes from cell culture supernatants and biological fluids. Curr. Protoc. Cell Biol. 30, 3.22.1-3.22.29 (2006).

78. Laulagnier, K. et al. Mast cell- and dendritic cell-derived exosomes display a specific lipid composition and an unusual membrane organization. Biochem. J. 380, 161-171 (2004)

79. Li, H. Minimap2: pairwise alignment for nucleotide sequences. Bioinformatics 34, 3094-3100 (2018)

\section{Acknowledgements}

We thank all of the patients involved in this study, as well as the dedicated medical and research staff who fight against SARS-CoV-2 worldwide; X. Liu from LipidALL Technologies for assistance with graphical illustrations, B. Jiang, S. Qi and H. Zhou for technical assistance. This work was financially supported by grants from the National Key R\&D Program of China to G.S. (2018YFA0800901, 2018YFA0506900), the National Natural Science Foundation of China to G.S. (31871194, 92057202), the Innovative Research Team in the National Natural Science Foundation of China to F.-S.W. (81721002) and the Youth Talent Lifting Project (2020-JCJQ-QT-034) to J.-W.S.

\section{Author contributions}

S.M.L., C.Z., J.-W.S., S.B. and G.S. conceived and designed the study, S.M.L., C.Z., Z.W., Z.N., S.Z., J.-W.S., J.L., M.M. and W.-J.C. carried out lipidomics and the majority of the molecular and cell experiments. S.Y., L.H., M.S., Z.X., F.-P.M., M.-J.Z. and L.S. collected clinical samples and curated clinical data under the supervision of F.Z. and F.-S.W. J.C. and J.W. conducted RNA-seq experiments. B. Lee analysed RNA-seq data. X.H. and Y.W. conducted proteomic experiments. L.M., G.H.C. and B. Li analysed proteomic and lipidomic data. G.S. directed the research and co-wrote the paper with S.M.L.

\section{Competing interests}

S.M.L., G.H.C. and B. Li are employees of LipidALL Technologies. The other authors declare no competing interests.

\section{Additional information}

Extended data is available for this paper at https://doi.org/10.1038/s42255-021-00425-4. Supplementary information The online version contains supplementary material available at https://doi.org/10.1038/s42255-021-00425-4.

Correspondence and requests for materials should be addressed to J.-W.S., F.Z., F.-S.W or G.S

Peer review information Nature Metabolism thanks Alberto Bartolome and the other, anonymous, reviewer(s) for their contribution to the peer review of this work. Primary Handling Editor: George Caputa.

Reprints and permissions information is available at www.nature.com/reprints.

Publisher's note Springer Nature remains neutral with regard to jurisdictional claims in published maps and institutional affiliations.

(C) The Author(s), under exclusive licence to Springer Nature Limited 2021 
a

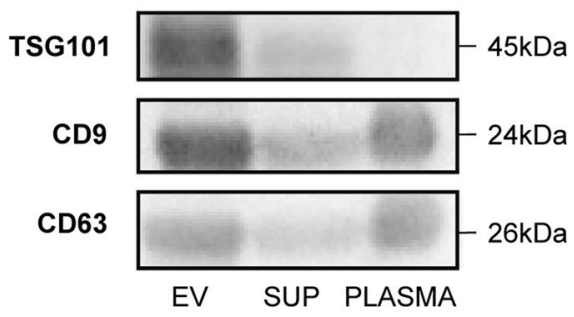

b Annexin A1

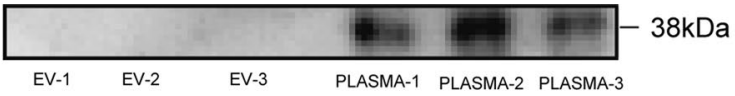

c

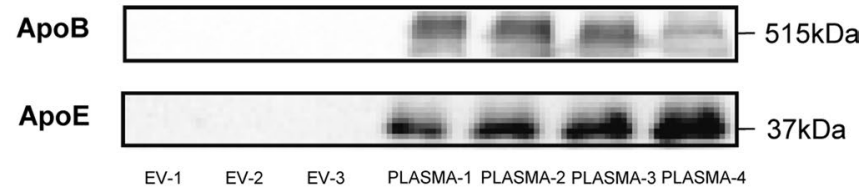

d

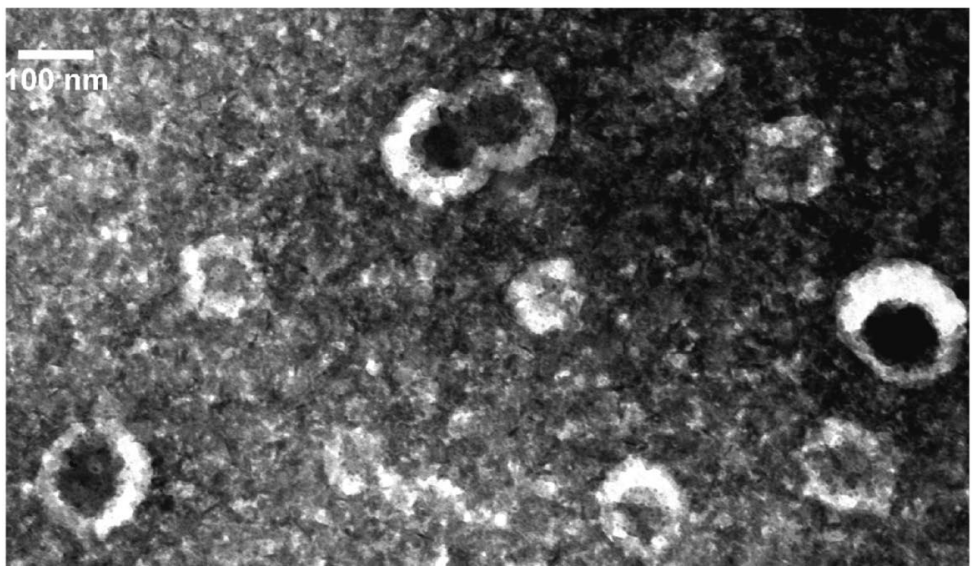

e

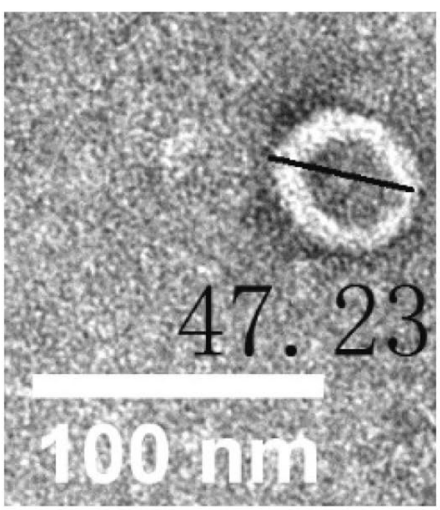

Extended Data Fig. 1 | Characterization of exosome-enriched EVs. a, Immunoblot analysis of exosome markers including TSG101, CD9 and CD63 in the isolated extracellular vesicle (EV) fractions relative to supernatant (SUP) and plasma (representative blot from $n=3$ independent experiments). b-c, Immunoblot analysis of microvesicle marker protein Annexin A1 (b) and lipoprotein marker proteins including apolipoprotein B (ApoB) and apolipoprotein $E(A p o E)$ (c) in EV protein fractions compared to plasma protein fractions (representative blot from $n=2$ independent experiments). Equal amount of total proteins ( $30 \mu \mathrm{g})$ was loaded onto each lane $(\mathbf{a}, \mathbf{b}, \mathbf{c})$. d, Wide-field transmission electron microscopy (TEM) image (negative staining) of isolated EV fractions at 10X dilution. e, Close-up electron microscopic image of a negatively stained EV at 500X dilution with estimated diameter $=47.23 \mathrm{~nm}$, which exhibited cup-shaped membrane vesicle morphology characteristic of exosomes. Representative images from $\mathrm{n}=2$ independent experiments $(\mathbf{d}, \mathbf{e})$. 


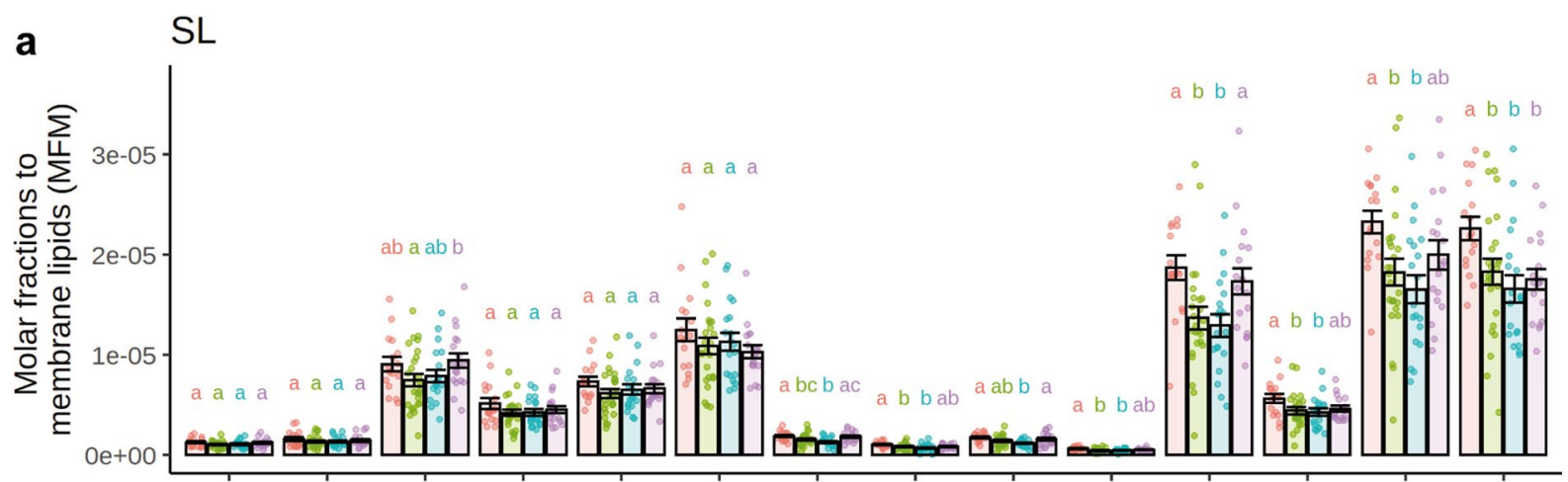

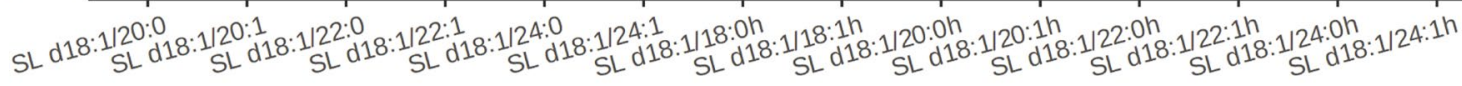

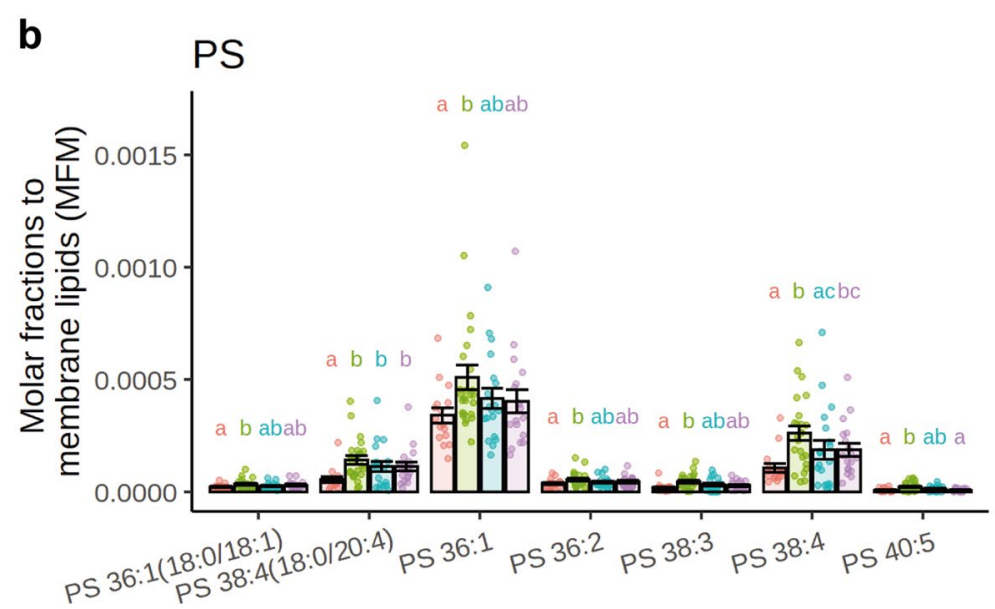

Extended Data Fig. 2 | Levels of sulfatides and phosphatidylserines in EVs. a,b, Barplots illustrating changes in individual species of sulfatides (SL) (a) and phosphatidylserines (PS) (b) across the four temporal stages of COVID-19 (S1: $n=16 ; S 2: n=25 ; S 3: n=19 ; S 4: n=18 ; n=$ number of patients). Data were presented as mean \pm SEM, P-value from two-sided Dunn's test for pair-wise comparison were indicated. $P<0.05$ was considered statistically significant. Statistical significance of all pair-wise comparisons were indicated using a letter-based representation, that two groups sharing a common letter are not statistically different at $\mathrm{P}<0.05$. 
a Free Cholesterol

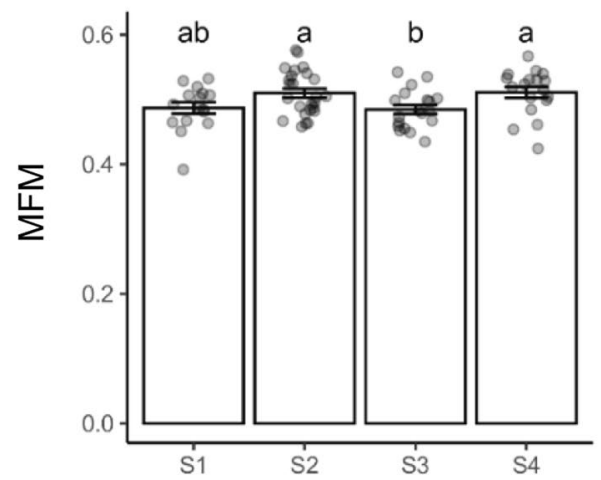

b $\quad \mathrm{TAG} /(\mathrm{DAG}+\mathrm{FFA})$

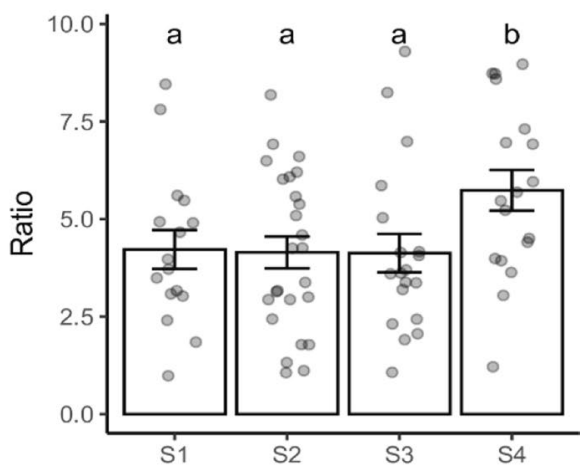

Extended Data Fig. 3 | Lipid changes in EVs from four temporal stages of COVID-19. a,b, Barplots illustrating changes in free cholesterols (a) and the ratios of triacylglycerols (TAGs) to their lipolytic products that is sum of diacylglycerols (DAGs) and free fatty acids (FFAs) (b) across four temporal stages of COVID-19 (S1: $n=16 ; S 2: n=25 ; S 3: n=19 ; S 4: n=18 ; n=$ number of patients). Data were presented as mean + SEM, P-value from two-sided Dunn's test for pair-wise comparison were indicated. $\mathrm{P}<0.05$ was considered statistically significant. Two groups sharing a common letter are not statistically different at $\mathrm{P}<0.05$ level. 
a

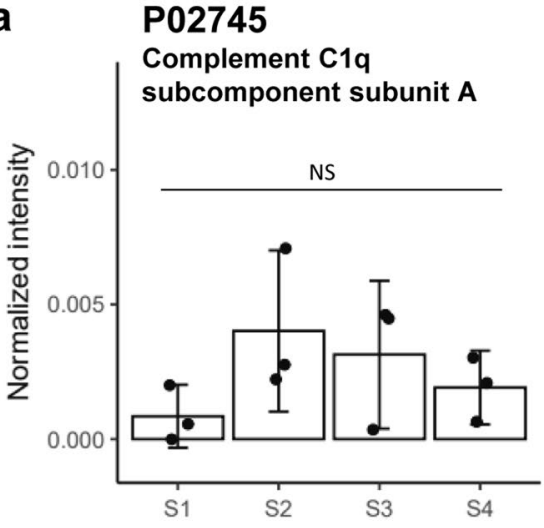

C

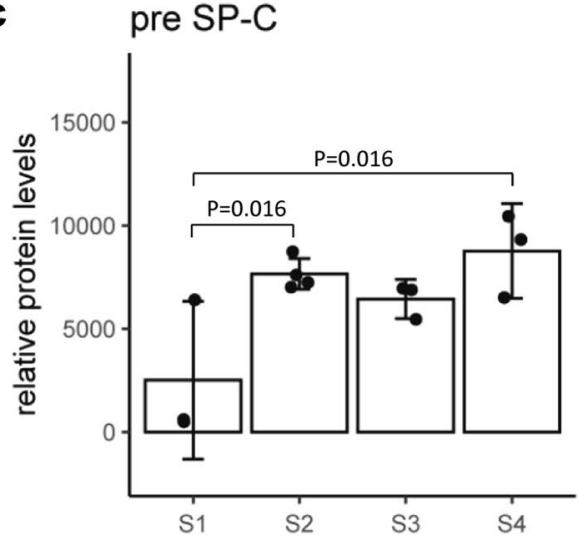

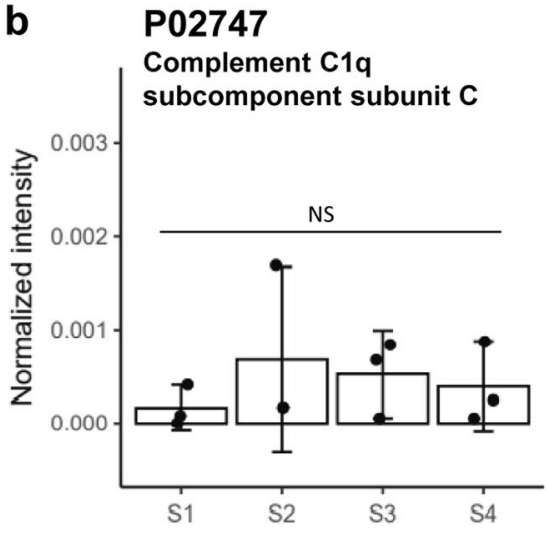

d

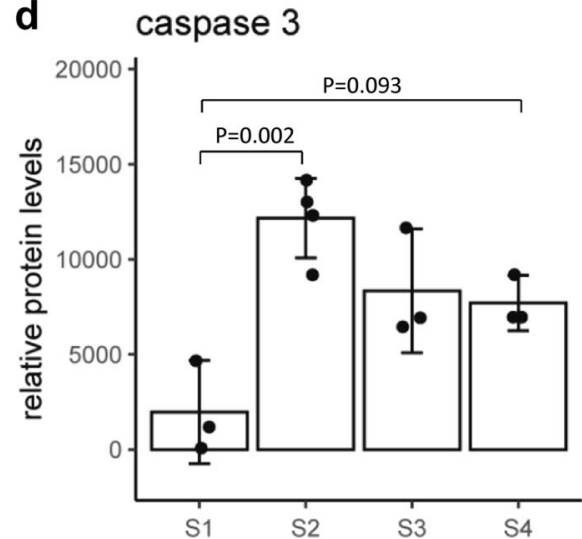

Extended Data Fig. 4 | Protein changes in EVs from four temporal stages of COVID-19. a,b, Levels of complement C1q subcomponent subunit A (a) and complement C1q subcomponent subunit $C(\mathbf{b})$ in EVs across four temporal stages of COVID-19 quantitated by proteomics ( $n=3$ independently pooled patient samples) were analysed for each clinical stage. P-value from two-sided Games-Howell's test for pair-wise comparisons were indicated. c-d, Immunoblot analyses of premature SP-C (c) and caspase 3 proenzyme (d) $(n=3$ for S1, S3, S4; $n=4$ for S2; $n=$ number of patients). Data were presented as mean + SEM. P-value from two-sided Dunn's test for pair-wise comparisons were indicated. $\mathrm{P}<0.05$ was considered statistically significant. NS: non-significant. 
a

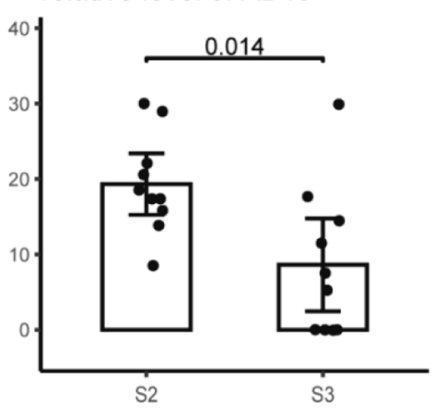

b

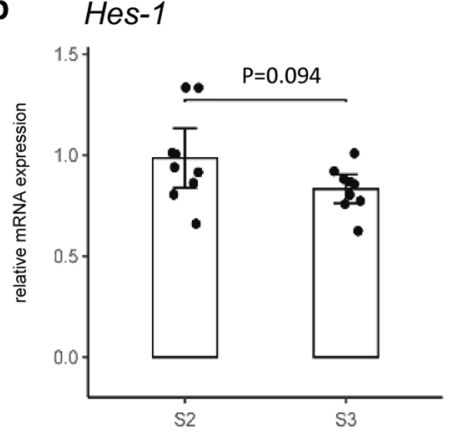

Hes-5

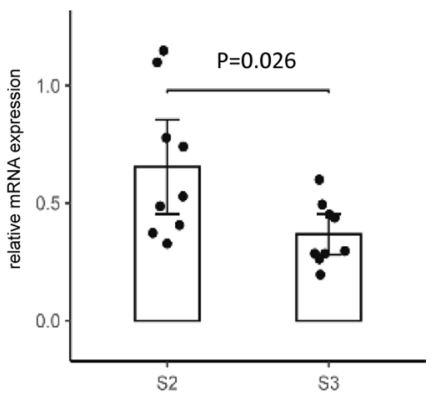

Extended Data Fig. 5 | Altered gamma-secretase activity in S2 and S3 phases of COVID-19. a, Barplot illustrates changes in the level of A $\beta 40$ in the exosome-free plasma of $S 2$ and $S 3$ patients ( $n=10$ for $S 2, n=10$ for $S 3 ; n=$ number of patients). The level of $A \beta 40$ was quantitated using sandwich ELISA (Invitrogen, KHB3481). Total protein was extracted from supernatant after exosome isolation from plasma, and equal amount of protein ( $30 \mu \mathrm{g}$ ) was added to each well on the ELISA plate for A $\beta 40$ quantitation. The absorbance was read off at $450 \mathrm{~nm}$ using a 96 -well plate reader, and the level of $A \beta 40$ in each sample was calculated from a standard curve. Each patient sample was analysed in duplicates and the mean value was plotted. Data were presented as mean + SEM, P-value from two-sided Wilcoxon's rank-sum test was displayed. b. Barplots on changes in the relative mRNA expression of Hes-1 and Hes-5 in $\mathrm{Hep} \overline{\mathrm{G}} 2$ cells treated with S2 EVs and S3 EVs, respectively. Data were presented as mean \pm SEM. P-value from two-sided Student's $t$ test was displayed $(n=9$ replicates from 3 independent cultures for each group). 

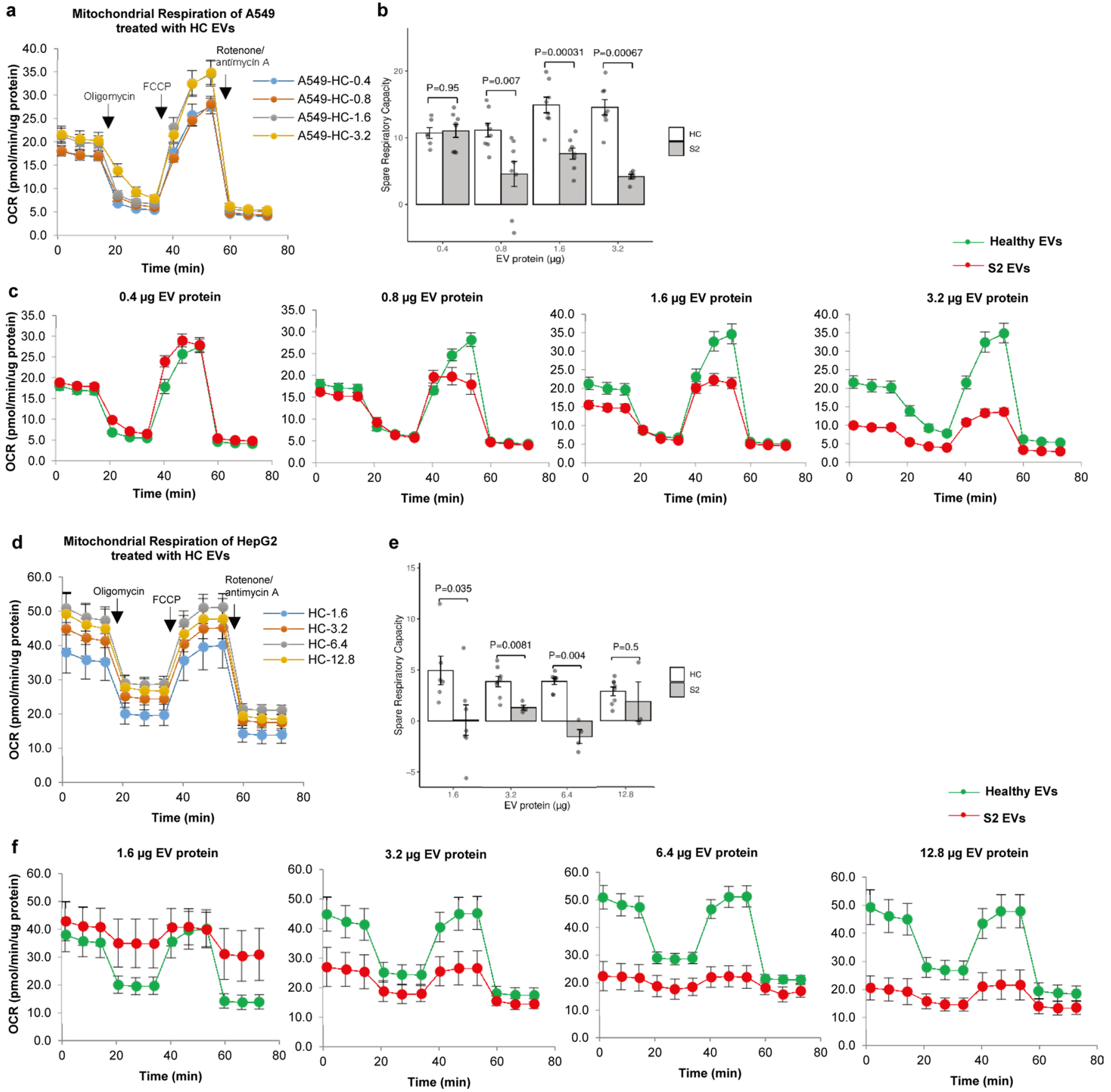

Extended Data Fig. 6 | Cellular metabolic analysis using the Seahorse XeF96 extracellular flux analyzer. $2 \times 10^{4}$ cells were seeded in each 96 -well and grown overnight prior to EV treatment. Mitochondrial respiration, as indicated by oxygen consumption rate (OCR) under basal conditions and with the addition of mitochondria poisons including oligomycin $(1 \mu \mathrm{M})$, carbonyl cyanide-4-(trifluoromethoxy) phenylhydrazone $(\mathrm{FCCP})(1 \mu \mathrm{M})$, rotenone $(1 \mu \mathrm{M})$ and antimycin $\mathrm{A}(1 \mu \mathrm{M})$ at indicated time points were measured. $\mathbf{a}, \mathbf{d}, \mathrm{OCR}$ curves of cells treated with increasing concentrations of HC EVs $(0.4-3.2 \mu \mathrm{g})$ for A549 cells (a) and (1.6-12.8 $\mathrm{gg}$ ) for HepG2 cells (d) showed that the experimental concentration range of HC EVs investigated did not trigger cellular stress response. b, e, Spare respiratory capacity of A549 cells (b) and HepG2 cells (e) treated with different concentrations of HC EVs compared to S2 EVs. P-values from two-sided Mann-Whitney U test was displayed $(\mathbf{b}, \mathbf{e})$. c, f, OCR curves of A549 cells (c) and HepG2 cells (f) treated with increasing concentrations HC EVs compared to S2 EVs. A final EV protein concentration at $3.2 \mu \mathrm{g}$ protein was selected for A549 cells and $6.4 \mu \mathrm{g}$ protein was selected for HepG2 cells. b, c on A549 cells: $n=6,8,8,8$ independent cultures for $0.4 \mu \mathrm{g}, 0.8 \mu \mathrm{g}, 1.6 \mu \mathrm{g}$ and $3.2 \mu \mathrm{g}$ of HC EV proteins, respectively; $\mathrm{n}=8,8,8$, 6 independent cultures for $0.4 \mu \mathrm{g}, 0.8 \mu \mathrm{g}, 1.6 \mu \mathrm{g}$ and $3.2 \mu \mathrm{g}$ of S2 EV proteins, respectively. e, $\mathbf{f}$ on HepG 2 cells: $\mathrm{n}=6,8,8,8$ independent cultures for $1.6 \mu \mathrm{g}, 3.2 \mu \mathrm{g}, 6.4 \mu \mathrm{g}$ and $12.8 \mu \mathrm{g}$ of HC EV proteins, respectively; $\mathrm{n}=7,4,4,3$ independent cultures for $1.6 \mu \mathrm{g}, 3.2 \mu \mathrm{g}, 6.4 \mu \mathrm{g}$ and $12.8 \mu \mathrm{g}$ of S2 EV proteins, respectively). Data were presented as mean \pm SEM. 


\section{Reporting Summary}

Nature Research wishes to improve the reproducibility of the work that we publish. This form provides structure for consistency and transparency in reporting. For further information on Nature Research policies, see our Editorial Policies and the Editorial Policy Checklist.

\section{Statistics}

For all statistical analyses, confirm that the following items are present in the figure legend, table legend, main text, or Methods section.

n/a Confirmed

$\square$ דhe exact sample size $(n)$ for each experimental group/condition, given as a discrete number and unit of measurement

$\square$ \A statement on whether measurements were taken from distinct samples or whether the same sample was measured repeatedly

$\square$ The statistical test(s) used AND whether they are one- or two-sided

$\square$ Only common tests should be described solely by name; describe more complex techniques in the Methods section.

$\square \bigotimes$ A description of all covariates tested

$\square$ \A description of any assumptions or corrections, such as tests of normality and adjustment for multiple comparisons

$\triangle \square$ A full description of the statistical parameters including central tendency (e.g. means) or other basic estimates (e.g. regression coefficient) AND variation (e.g. standard deviation) or associated estimates of uncertainty (e.g. confidence intervals)

$\square$ For null hypothesis testing, the test statistic (e.g. $F, t, r$ ) with confidence intervals, effect sizes, degrees of freedom and $P$ value noted Give $P$ values as exact values whenever suitable.

Х $\square$ For Bayesian analysis, information on the choice of priors and Markov chain Monte Carlo settings

Х $\square$ For hierarchical and complex designs, identification of the appropriate level for tests and full reporting of outcomes

$\bigotimes \square$ Estimates of effect sizes (e.g. Cohen's $d$, Pearson's $r$ ), indicating how they were calculated

Our web collection on statistics for biologists contains articles on many of the points above.

\section{Software and code}

Policy information about availability of computer code

Data collection Analyst (v1.6.3), XCalibur(v4.1),ONT MinKNOW software (v19.10.1), Guppy (v3.2.4)

Data analysis Analyst (v1.6.3), R (v4.0.2), R (v3.6.2), Bioconductor (v3.10), ClusterProfiler (v3.14.3), org.hs.eg.db (v3.10.0), Thermo Scientific Proteome DiscovererTM software(v2.3), Python (v2.7), Perl (v5.2), Apogee Histogram Software (v255.0.0.263), Qcat(v1.1.0), minimap2 (v2.17), BEDtools multicov(v2.27.1), ComplexHeatmap (v2.7.10.9001), circlize (v0.4.12), ggplot2 (v3.3.3), DESeq2 (v1.30.1), Hmisc (v4.4-2), circlize (v0.4.12), chorddiag (v0.1.2)

For manuscripts utilizing custom algorithms or software that are central to the research but not yet described in published literature, software must be made available to editors and reviewers. We strongly encourage code deposition in a community repository (e.g. GitHub). See the Nature Research guidelines for submitting code \& software for further information.

\section{Data}

Policy information about availability of data

All manuscripts must include a data availability statement. This statement should provide the following information, where applicable:

- Accession codes, unique identifiers, or web links for publicly available datasets

- A list of figures that have associated raw data

- A description of any restrictions on data availability

Proteomics data of exosome-enriched EVs have been deposited to the ProteomeXchange Consortium via the PRIDE partner repository with the dataset identifier PXD026091. RNAseq data of EV-treated cells have been deposited to the GEO omnibus under the accession number GSE174668. Uniprot database is accessible via www.uniprot.org. KEGG pathway database for proteomics data analysis is accessible via http://www.genome.jp/kegg/pathway.html. Reactome and KEGG pathway databases for RNAseq data analysis is accessible at MSigDB (version 7.3) via http://www.gsea-msigdb.org/gsea/msigdb/. Minimal datasets including lipidomics, 


\section{Field-specific reporting}

Please select the one below that is the best fit for your research. If you are not sure, read the appropriate sections before making your selection.

$\searrow$ Life sciences

Behavioural \& social sciences

Ecological, evolutionary \& environmental sciences

For a reference copy of the document with all sections, see nature.com/documents/nr-reporting-summary-flat.pdf

\section{Life sciences study design}

All studies must disclose on these points even when the disclosure is negative.

Sample size No sample size calculation was performed. We included all available samples that met our clinical classification scheme collected in the two hospitals designated for COVID-19 patients in Beijing, between January to July 2020.

Data exclusions If more than one blood samples from the same patient were classified under the same clinical stage, repeated samples were excluded to ensure that all blood samples in each clinical stage came from distinct subjects.

Replication All experiments were conducted with biological replicates. For lipidome analysis of exosome, the same size were $n=16, n=23, n=19, n=18$ distinct patients for each clinical stage. For proteomic analysis, due to the limiting protein quantity remaining after lipid extraction, samples were pooled for proteomics, and each clinical stage contains 3 independently pooled samples (i.e. $n=3$ for each clinical stage). For Western Blot analysis, $n=3$ or 4 distinct patients for each clinical stage. For exosome-treated cells, $n=3$ independent cultures for each treatment condition. Experiments were repeated as detailed in the figure legends. All attempts at replication were successful.

Randomization Patient allocation into each of the four clinical stage were not random and were based on selection criteria including blood sample collection date with respect to hospital admission and discharge dates, SARS-COV-2 RNA test result, and associated laboratory indices on the date of collection, as detailed under Study Design and Participants. The order of samples were randomized at metabolite extraction steps and LCMS runs. One QC sample is inserted every ten samples.

Blinding Clinicians performing blood sample collection and experimenters performing blood sample processing, metabolite extraction and LCMS analyses were blinded from the sample grouping used in this study. Data scientists were not blinded at the stage of data analysis because the results were quantitative and did not require subjective judgment nor interpretation.

\section{Reporting for specific materials, systems and methods}

We require information from authors about some types of materials, experimental systems and methods used in many studies. Here, indicate whether each material, system or method listed is relevant to your study. If you are not sure if a list item applies to your research, read the appropriate section before selecting a response.

Materials \& experimental systems

\begin{tabular}{l|l}
\hline$n / a$ & Involved in the study \\
$\square$ Antibodies
\end{tabular}

\begin{tabular}{l|l} 
Methods \\
\hline n/a & Involved in the study \\
\hline & $\square$ ChIP-seq \\
$\bigotimes$ & $\square$ Flow cytometry \\
$\bigotimes$ & $\square$ MRI-based neuroimaging
\end{tabular}

\section{Antibodies}

Primary antibodies for western blot:

Presenilin 1(Abcam, cat\# ab76083, clone: EP2000Y, lot\# GR3237647-2, dilution 1:2000)

CD9(C-4)(Santa Cruz, cat\# sc-13118, clone: C-4, lot\# 10619, dilution 1:500)

CD63(Santa Cruz, cat\# sc-5275, clone: MX-49.129.5, lot\# A2220, dilution 1:500)

TSG101(Abcam, cat\# ab125011, clone: EPR7130(B), lot\# GR299332-32, dilution 1:2500)

SP-C(Santa Cruz, cat\# sc-13979, clone: FL-197, dilution 1:500)

IL-6(Abcam, cat\# ab233706, clone: EPR21711, lot\# GR3362534-5, dilution 1:2000)

Caspase-3(CST, cat\# 9662, lot\# 18, dilution 1:2000)

Annexin A1(CST, cat\# 32934, clone: D5V2T, lot\# 1, dilution 1:1000)

ApoB(Merck Millipore, cat\# AB742, lot\# 2957417, dilution 1:3000)

ApoE(Merck Millipore, cat\# AB947, dilution 1:1000) 
Secondary antibodies for western blot:

Horseradish peroxidase-coupled Goat anti-Mouse IgG secondary antibody(ZSGB-BIO, cat\# ZB-2301, lot\#206791028, dilution 1:5000) Horseradish peroxidase-coupled Goat anti-Rabbit IgG secondary antibody(ZSGB-BIO, cat\# ZB-2305, lot\#200061221, dilution 1:5000) Peroxidase Conjugated, H+L Rabbit Anti-Goat IgG secondary antibody(Biosharp, cat\# BL004A, lot\#6801015, dilution 1:2500)

All antibodies were bought from commercial vendors and specificity can be retrieved from the manufacturer's website listed below, we also included an example literature from the manufacturer's website supporting the validation of the stated antibody:

Presenilin 1(Abcam,ab76083) (27 citations)

https://www.citeab.com/antibodies/748416-ab76083-anti-presenilin-1-ps-1-antibody-ep2000y?des=54972a187dfc417f

The presenilin-1 antibody is knockout-validated by Abcam, one of the most accepted and trusted validation processes for antibody specificity using human KO haploid cell lines generated via CRISPR-Cas9 and certified via Sanger sequencing. This type of KO cell lines provides a complete loss-of-function phenotype from a single allele $\mathrm{KO}$ and eliminates any masking of the knockout from a second allele seen in diploid cell models. More information at https://www.abcam.com/primary-antibodies/knockout-validation.

CD9 (C-4)(Santa Cruz, sc-13118)(104 citations)

https://www.citeab.com/antibodies/810356-sc-13118-cd9-antibody-c-4?des=c1508687577f6be3

The antibody was validated in human exosomes in published literature (PMID: 30065664)

CD63 (Santa Cruz, sc-5275) (191 citations)

https://www.citeab.com/antibodies/810017-sc-5275-cd63-antibody-mx-49-129-5?des=cbac89686f324477

The antibody was validated in human exosomes in published literature (PMID: 31858766 )

TSG101 (Abcam, ab125011) (168 citations)

https://www.citeab.com/antibodies/757168-ab125011-anti-tsg101-antibody-epr7130-b?des=5c15d14485513a16

The antibody was validated in human exosomes in published literature (PMID: 33100957)

IL-6 (Abcam, ab233706) (5 citations)

https://www.citeab.com/antibodies/5061036-ab233706-anti-il-6-antibody-epr21711?des=bade498507e0b69a

The IL- 6 antibody is knockout-validated by Abcam, one of the most accepted and trusted validation processes for antibody specificity using human KO haploid cell lines generated via CRISPR-Cas9 and certified via Sanger sequencing. This type of KO cell lines provides a complete loss-of-function phenotype from a single allele $\mathrm{KO}$ and eliminates any masking of the knockout from a second allele seen in diploid cell models. More information at https://www.abcam.com/primary-antibodies/knockout-validation.

Caspase-3(CST, 9662) (2663 citations)

https://www.citeab.com/antibodies/126298-9662-caspase-3-antibody?des=d2b21d9c073df1df

The Caspase- 3 antibody is knockout-validated by CST.

Annexin A1(CST,32934) (9 citations)

https://www.citeab.com/antibodies/3394219-32934-annexin-a1-d5v2t-xp-rabbit-mab?des=7d2def4aeba584fd

The antibody was validated in human cancer cell exosomes in published literature (PMID: 32720716)

ApoB(Merck Millipore,AB742) (31 citations)

https://www.citeab.com/antibodies/224078-ab742-anti-apolipoprotein-b-antibody?des=90c71aOfcdb9fef1

The antibody was validated in human plasma in published literature (PMID: 32737203)

ApoE(Merck Millipore,AB947) (86 citations)

https://www.citeab.com/antibodies/224356-ab947-anti-apolipoprotein-e-antibody?des=6a5e7486bf5c21c7

The antibody was validated in human stem cells in published literature (PMID: 28132833)

SP-C(Santa Cruz,sc-13979) (31 citations)

https://www.scbt.com/p/sp-c-antibody-fl-197?requestFrom=search

The antibody was validated in human cancer cells in published literature (PMID: 23928700)

\section{Eukaryotic cell lines}

Policy information about cell lines

Cell line source(s)

HepG2 and A549 cell line obtained from ATCC

Authentication

Confirmed by morphological observation

Mycoplasma contamination

All cell lines were routinely tested for mycoplasma by PCR. All cell lines were negative.

Commonly misidentified lines

(See ICLAC register)

No commonly misidentified cell lines were used. 
Policy information about studies involving human research participants

Population characteristics

The reported confounders in the susceptibility to COVID-19 include age, sex and BMI. These clinical data were provided in Table 1 and Supplementary Data (excel, worksheet 1). No statistical significance in age, sex and BMI were noted in the samples across the four temporal stages of COVID-19 in our study.

Recruitment

All COVID-19 patients admitted to the designated hospitals with blood samples satisfying our clinical classification scheme, and with available clinical data records and SARS-CoV-2 RNA results were included into our study. No self-selection bias.

Ethics oversight

This study was approved by the ethics committee of the Fifth Medical Center of the PLA General Hospital and written informed consent was obtained from all subjects enrolled in this study.

Note that full information on the approval of the study protocol must also be provided in the manuscript. 